\title{
Bispecific IgG neutralizes SARS-CoV-2 variants and prevents escape in mice
}

https://doi.org/10.1038/s41586-021-03461-y

Received: 7 January 2021

Accepted: 16 March 2021

Published online: 25 March 2021

Check for updates

\author{
Raoul De Gasparo ${ }^{1,16}$, Mattia Pedotti ${ }^{1,16}$, Luca Simonelli', Petr Nickl ${ }^{2}$, Frauke Muecksch ${ }^{3}$, \\ Irene Cassaniti ${ }^{4}$, Elena Percivalle ${ }^{4}$, Julio C. C. Lorenzi ${ }^{5}$, Federica Mazzola', Davide Magri ${ }^{6}$, \\ Tereza Michalcikova ${ }^{2}$, Jan Haviernik ${ }^{7}$, Vaclav Honig ${ }^{7,8}$, Blanka Mrazkova ${ }^{2}$, Natalie Polakova ${ }^{2}$, \\ Andrea Fortova ${ }^{7}$, Jolana Tureckova ${ }^{2}$, Veronika latsiuk ${ }^{2}$, Salvatore Di Girolamo', Martin Palus ${ }^{7,8}$, \\ Dagmar Zudova ${ }^{2}$, Petr Bednar, ${ }^{7,9}$, Ivana Bukova ${ }^{2}$, Filippo Bianchini', Dora Mehn ${ }^{6}$, \\ Radim Nencka ${ }^{10}$, Petra Strakova ${ }^{7}$, Oto Pavlis ${ }^{11}$, Jan Rozman ${ }^{2}$, Sabrina Gioria ${ }^{6}$, \\ Josè Camilla Sammartino ${ }^{4}$, Federica Giardina ${ }^{4}$, Stefano Gaiarsa ${ }^{4}$, Qiang Pan-Hammarström ${ }^{12}$, \\ Christopher O. Barnes ${ }^{13}$, Pamela J. Bjorkman ${ }^{13}$, Luigi Calzolai ${ }^{6}$, Antonio Piralla ${ }^{4}$, \\ Fausto Baldanti ${ }^{4,14}$, Michel C. Nussenzweig ${ }^{5,15}$, Paul D. Bieniasz ${ }^{3,15}$, Theodora Hatziioannou ${ }^{3}$, \\ Jan Prochazka ${ }^{2}$, Radislav Sedlacek ${ }^{2}$, Davide F. Robbiani ${ }^{1 凶}$, Daniel Ruzek ${ }^{7,8 凶}$ \& Luca Varani $^{1 \bowtie}$
}

\begin{abstract}
Neutralizing antibodies that target the receptor-binding domain (RBD) of the SARS-CoV-2 spike protein are among the most promising approaches against COVID-19 ${ }^{1,2}$. A bispecific IgG1-like molecule (CoV-X2) has been developed on the basis of $\mathrm{C} 121$ and $\mathrm{C} 135$, two antibodies derived from donors who had recovered from COVID-19 ${ }^{3}$. Here we show that CoV-X2 simultaneously binds two independent sites on the RBD and, unlike its parental antibodies, prevents detectable spike binding to the cellular receptor of the virus, angiotensin-converting enzyme 2 (ACE2). Furthermore, CoV-X2 neutralizes wild-type SARS-CoV-2 and its variants of concern, as well as escape mutants generated by the parental monoclonal antibodies. We also found that in a mouse model of SARS-CoV-2 infection with lung inflammation, $\mathrm{CoV}-\mathrm{X} 2$ protects mice from disease and suppresses viral escape. Thus, the simultaneous targeting of non-overlapping RBD epitopes by IgG-like bispecific antibodies is feasible and effective, and combines the advantages of antibody cocktails with those of single-molecule approaches.
\end{abstract}

The COVID-19 pandemic has prompted substantial efforts to develop effective countermeasures against SARS-CoV-2. Preclinical data and phase-III clinical studies indicate that monoclonal antibodies could be effectively deployed for prevention or treatment during the viral symptoms phase of the disease $\mathrm{e}^{1,2}$. Cocktails of two or more monoclonal antibodies are preferred over a single antibody as these cocktails result in increased efficacy and the prevention of viral escape. However, this approach requires increased manufacturing costs and volumes, which are problematic at a time when the supply chain is under pressure to meet the high demand for COVID-19 therapeutic agents, vaccines and biologics in general ${ }^{4}$. Cocktails also complicate formulation ${ }^{5,6}$ and hinder strategies such as antibody delivery by viral vectors or by nonvectored nucleic acids ${ }^{7,8}$. One alternative is to use multispecific antibodies, which have the advantages of cocktails and single-molecule strategies.

To this end, we used structural information ${ }^{9}$ and computational simulations to design bispecific antibodies that would simultaneously bind to (i) independent sites on the same RBD and (ii) distinct RBDs on a spike (S) trimer. We evaluated several designs using atomistic molecular dynamics simulations, and produced four constructs: of these, CoV-X2 was the most potent neutralizer of SARS-CoV-2 pseudovirus, and had a half-maximal inhibitory concentration $\left(\mathrm{IC}_{50}\right)$ of $0.04 \mathrm{nM}$ (5.8 $\left.\mathrm{ng} \mathrm{ml}^{-1}\right)$ (Extended Data Fig. 1). CoV-X2 is a human-derived IgG1-like bispecific antibody in the CrossMAb format ${ }^{10}$ that is the result of the combination of the Fragment antigen binding ( $\mathrm{Fab}$ ) of the monoclonal antibodies $\mathrm{C} 121$ and $\mathrm{C} 135$, which are two potent neutralizers of SARS-CoV- ${ }^{3}$. Structural predictions showed that CoV-X2-but not its parental monoclonal antibodies-can bind bivalently to all RBD conformations on the S trimer, which prevents the binding of ACE2 receptor $^{11}$ (Fig. 1a, Extended Data Fig. 2).

CoV-X2 bound at a low nanomolar affinity to the RBD and S trimer of wild-type SARS-CoV-2 and to those of several naturally occurring SARS-CoV-2 variants, including B.1 (which contains D614G, in the S),

${ }^{1}$ Institute for Research in Biomedicine, Università della Svizzera italiana (USI), Bellinzona, Switzerland. ${ }^{2}$ Czech Centre of Phenogenomics and Laboratory of Transgenic Models of Diseases, Institute of Molecular Genetics of the Czech Academy of Sciences, Vestec, Czech Republic. ${ }^{3}$ Laboratory of Retrovirology, The Rockefeller University, New York, NY, USA. ${ }^{4}$ Molecular Virology Unit, Microbiology and Virology Department, Fondazione IRCCS Policlinico San Matteo, Pavia, Italy. ${ }^{5}$ Laboratory of Molecular Immunology, The Rockefeller University, New York, NY, USA.

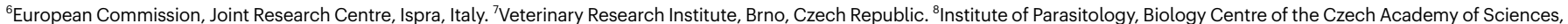
Ceske Budejovice, Czech Republic. ${ }^{9}$ Faculty of Science, University of South Bohemia, Ceske Budejovice, Czech Republic. ${ }^{10}$ Institute of Organic Chemistry and Biochemistry of the Czech Academy of Sciences, Prague, Czech Republic. ${ }^{11}$ Center of Biological Defense, Military Health Institute, Military Medical Agency, Techonin, Czech Republic. ${ }^{12}$ Department of Biosciences and Nutrition, Karolinska Institutet, Huddinge, Sweden. ${ }^{13}$ Division of Biology and Biological Engineering, California Institute of Technology, Pasadena, CA, USA. ${ }^{14}$ Department of Clinical Surgical Diagnostic and Pediatric Sciences, Università degli Studi di Pavia, Pavia, Italy. ${ }^{15}$ Howard Hughes Medical Institute, The Rockefeller University, New York, NY, USA. ${ }^{16}$ These authors contributed equally: Raoul De Gasparo, Mattia Pedotti.凶e-mail: drobbiani@irb.usi.ch; ruzekd@paru.cas.cz; luca.varani@irb.usi.ch 

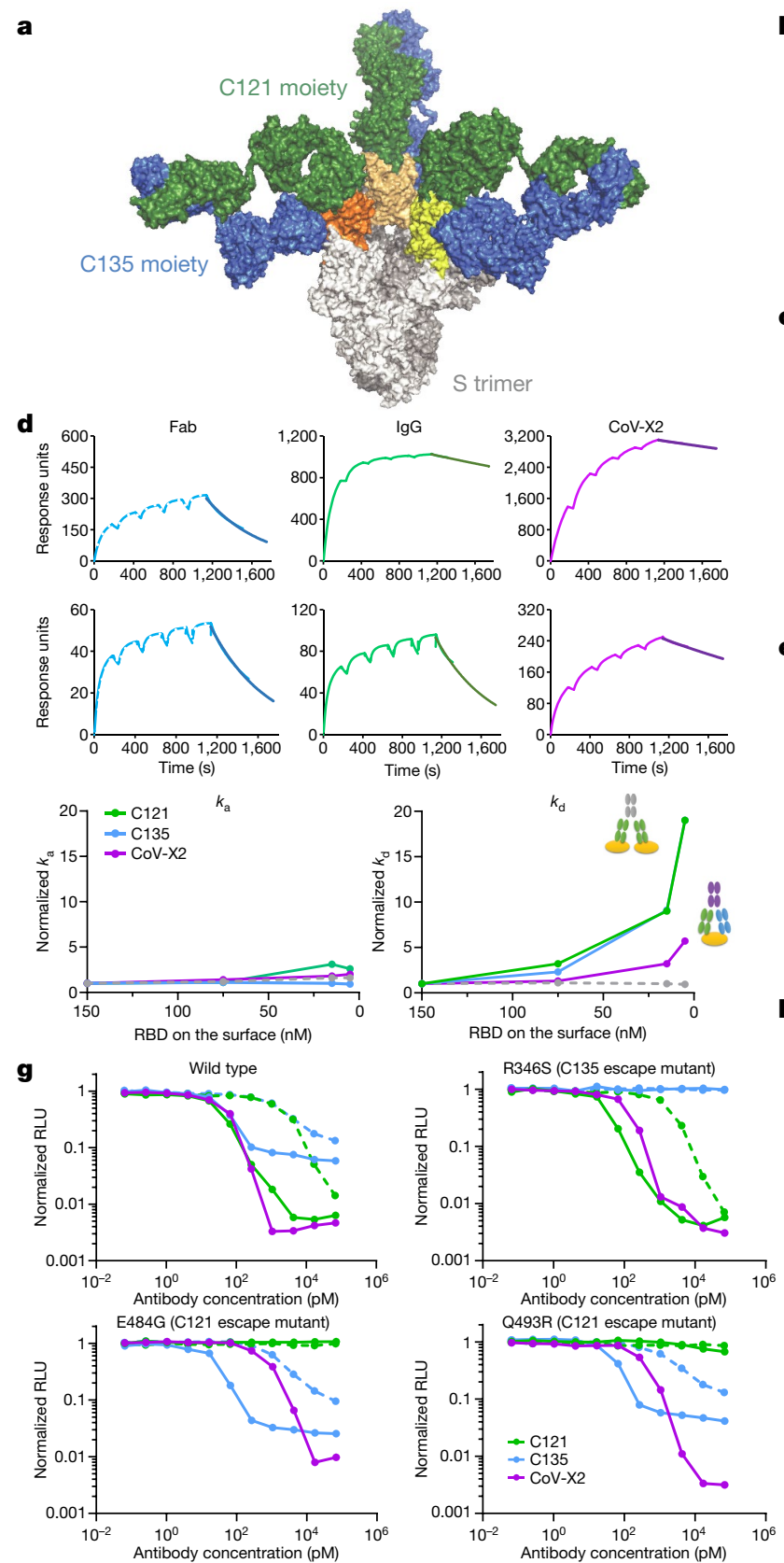

Fig. 1 | Biochemical and in vitro neutralizing properties of CoV-X2 are superior to those of its parental monoclonal antibodies. $a$, Computational simulations predict bivalent binding of $\mathrm{CoV}-\mathrm{X} 2$ to all three RBDs on the $\mathrm{S}$ trimer (Extended Data Fig. 2). Green and blue, $\mathrm{C} 121$ and $\mathrm{C} 135$ moieties, respectively; yellow and orange, RBDs.b, c, Surface plasmon resonance (SPR) demonstrates that both arms of $\mathrm{CoV}-\mathrm{X} 2$ are functional. In $\mathbf{b}$, immobilized RBD complexed with C121 (left) or C135 (right) (first antibody) binds to CoV-X2 (second antibody). In c, RBD-CoV-X2 prevents binding by $\mathrm{C} 121$ (left) or $\mathrm{C} 135$ (right) single monoclonal antibodies. Paler colours denote controls (second antibody only). d, Both arms of CoV-X2 bind simultaneously to the RBD, as avidity is retained at decreasing RBD concentrations. Top and middle, representative SPR traces indicating the dissociations of Fab (left), IgG (centre) or CoV-X2 (right) binding to RBD immobilized at $150 \mathrm{nM}$ (top) or $15 \mathrm{nM}$ (middle) on the SPR chip (Extended Data Fig. 6). Bottom, plots of normalized $k_{\mathrm{a}}$ (left) and $k_{\mathrm{d}}$ (right) obtained with different concentrations of immobilized RBD. Increasing normalized $k_{\mathrm{d}}$ values indicate loss of avidity. Solid lines, IgG; dotted lines, Fab. e, f, CoV-X2 prevents ACE2 binding to S trimer in an ELISA. ACE2 binding to

B.1.1.7 (which contains N501Y, in the RBD) and B.1.351 (which contains $\mathrm{K} 417 \mathrm{~N} / \mathrm{E} 484 \mathrm{~K} / \mathrm{N} 501 \mathrm{Y}$, in the RBD $)^{12,13}$, as well as to escape mutants generated by the parental monoclonal antibodies ${ }^{14}$ (Extended Data Figs. 3-5).
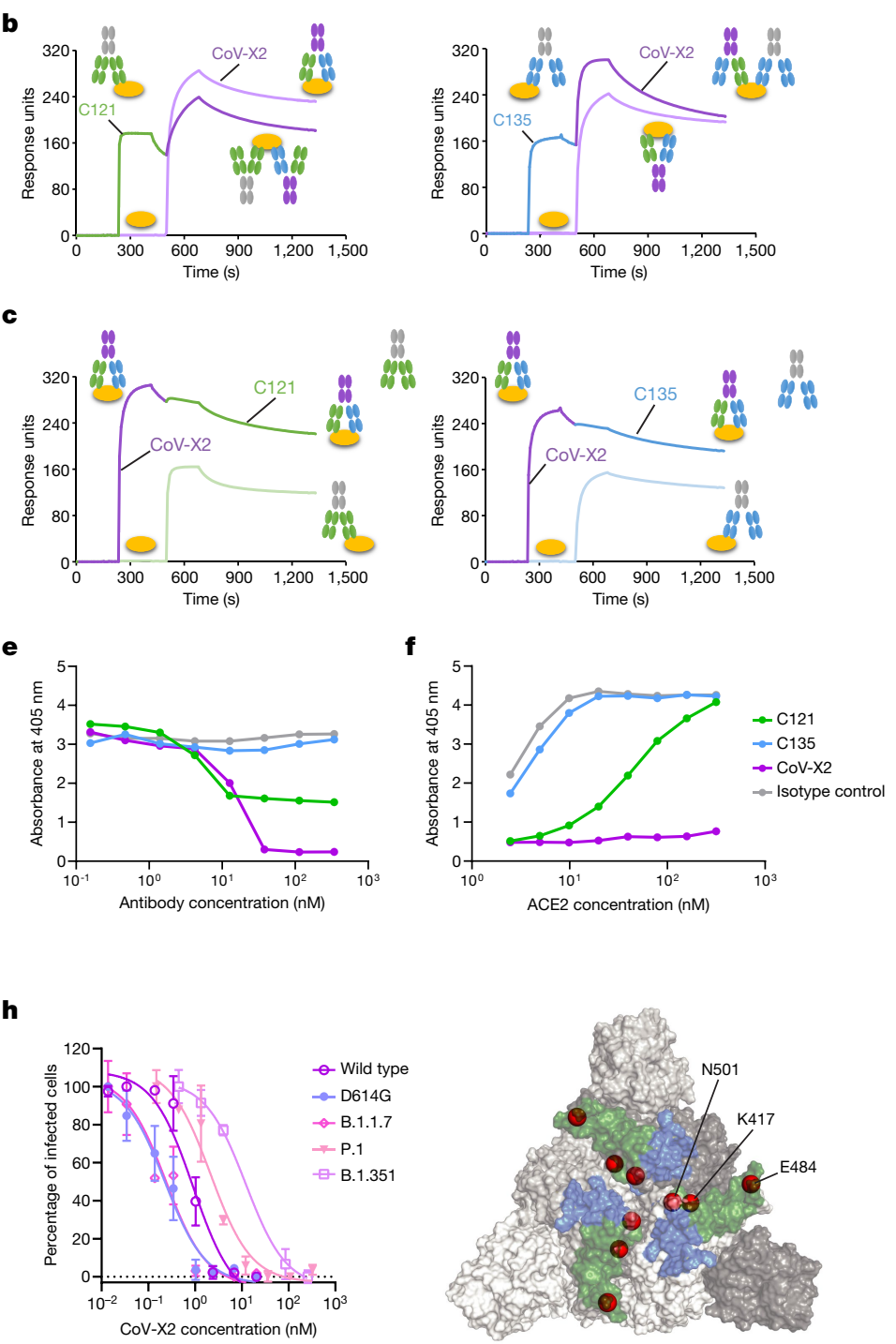

\begin{tabular}{c|ccc} 
& \multicolumn{3}{|c}{ RBD mutations } \\
& 417 & 484 & 501 \\
\hline Wild type & $\mathrm{K}$ & $\mathrm{E}$ & $\mathrm{N}$ \\
B.1.1.7 & $\mathrm{K}$ & $\mathrm{E}$ & $\mathrm{Y}$ \\
P.1 & $\mathrm{T}$ & $\mathrm{K}$ & $\mathrm{Y}$ \\
B.1.351 & $\mathrm{N}$ & $\mathrm{K}$ & $\mathrm{Y}$
\end{tabular}

antibody-S-trimer complexes measured with increasing concentration of indicated antibody and constant ACE2 (e), or at constant antibody concentration with increasing ACE2 (f). Mean of two replicates shown. g, CoV-X2 neutralizes SARS-CoV-2 pseudovirus and escape mutants generated by its parental monoclonal antibodies. Normalized relative luminescence (RLU) for cell lysates after infection with NanoLuc-expressing SARS-CoV-2 pseudovirus in the presence of increasing concentrations of antibodies. Wild-type SARS-CoV-2 pseudovirus (top left) is shown alongside an escape mutant generated in the presence of $\mathrm{C} 135$ (R346S; top right), or two escape mutants generated in the presence of C121 (E484G (bottom left) and Q493R (bottom right) $)^{14}$. Dashed lines, parental Fabs; solid lines, IgG. Mean of two independent experiments with two replicates each. $\mathbf{h}$, Neutralization of SARS-CoV-2 isolates with sequences corresponding to wild-type virus (which was first isolated in China), and the B.1(D614G), B.1.1.7, P.1 and B.1.351 variants. Mean of three experiments with s.d. RBD residues mutated in the variants are indicated in the table and as red spheres on the $S$ trimer structure, on which the epitopes of C135 (blue) and C121 (green) are shown.

CoV-X2 also bound to preformed C121-RBD and C135-RBD complexes, which confirmed that both of its arms are functional (Fig. 1b, c). Next, we used an avidity assay by surface plasmon resonance to 
experimentally confirm the computational prediction that $\mathrm{CoV}-\mathrm{X} 2 \mathrm{can}$ simultaneously engage two sites on the same RBD (Fig. 1d, Extended Data Fig. 6, Methods). Avidity occurs when IgG binds bivalently to antigens, which results in slower dissociation rates $\left(k_{\mathrm{d}}\right)$ (Extended Data Fig. 6a). Accordingly, $\mathrm{C} 121$ and $\mathrm{C} 135 \mathrm{IgG}$ showed avidity at high concentrations of antigen, owing to the intermolecular binding of adjacent RBDs; at lower concentrations of antigen, the $k_{\mathrm{d}}$ was faster as intermolecular binding was prevented by the increased distance between RBD molecules, which resulted in loss of avidity. Intramolecular avidity is not possible for $\mathrm{C} 121$ and $\mathrm{C} 135$ because a single epitope is available on each RBD molecule. By contrast, CoV-X2 maintained avidity even at low concentrations of antigen, which indicates that it undergoes bivalent, intramolecular binding (Fig. 1d, Extended Data Fig. 6). We then performed enzyme-linked immunosorbent assays (ELISAs) to evaluate the ability of CoV-X2 to inhibit the binding of recombinant ACE2 to the S trimer (Fig. 1e, f). Consistent with the structural information ${ }^{9}, \mathrm{C} 135$ did not affect the interaction between ACE2 and S. C121, which occupies the ACE2-binding site on the RBD, prevented ACE2 binding only partially. By contrast, ACE2 binding was not detected in the presence of $\mathrm{CoV}-\mathrm{X} 2$, which suggests a synergistic effect by the two moieties that comprise the bispecific antibody.

To assess the neutralizing ability of CoV-X2 in vitro, we first used SARS-CoV-2 pseudoviruses ${ }^{15}$. The bispecific antibody neutralized pseudovirus that carries wild-type SARS-CoV-2S at sub-nanomolar concentrations $\left(\mathrm{IC}_{50}=0.04 \mathrm{nM}\left(5.8 \mathrm{ng} \mathrm{ml}^{-1}\right)\right.$ and $90 \%$ inhibitory concentration of $\left.0.3 \mathrm{nM}\left(44 \mathrm{ng} \mathrm{ml}^{-1}\right)\right)$, which was similar to or better than the parental IgG and an over 100 -fold-better IC $_{50}$ than that of the parental Fabs (Fig. 1g). CoV-X2 remained effective against pseudoviruses that bear escape mutations that make them resistant to the individual monoclonal antibodies ${ }^{14}$ (Fig. 1g), and against a pseudovirus with RBD mutations that are found in the B.1.351 variant (which was first reported in South Africa) $\left(\mathrm{IC}_{50}=1.3 \mathrm{nM}\left(191 \mathrm{ng} \mathrm{ml}^{-1}\right)\right)$ (Extended Data Fig. 5). To confirm the efficacy of CoV-X2, we performed plaque-reduction neutralization assays with infectious virus. CoV-X2 efficiently neutralized wild-type SARS-CoV-2 $\left(\mathrm{IC}_{50}=0.9 \mathrm{nM}\right)$; the B.1 variant that carries S(D614G), which was first detected in Europe $\left(\mathrm{IC}_{50}=0.2 \mathrm{nM}\right)$; the B.1.1.7 variant, which was first observed in the UK $\left(\mathrm{IC}_{50}=0.2 \mathrm{nM}\right)$; the P.1 variant, which was first isolated in Brazil $\left(\mathrm{IC}_{50}=2.1 \mathrm{nM}\right)$; and the B.1.351 variant $\left(\mathrm{IC}_{50}=12 \mathrm{nM}\right)$ (Fig. 1h). The P.1 and B.1.351 variants have almost identical mutations in the RBD; the only difference is the presence of an Asn (in B.1.351) versus Thr (in P.1) at position 417, which does not interact with CoV-X2. Nonetheless, the neutralization of B.1.351 was lower than that for P.1, which suggests that there are either some conformational differences in the RBD between the two variants or long-range effects that derive from additional mutations in the $\mathrm{S}$. A similar behaviour is seen with the wild-type sequence (D614), which has a lower neutralization than $\mathrm{S}$ (D614G) even if no other difference is present; a plausible explanation is that a Gly at position 614 makes the $\mathrm{CoV}$-X2 epitopes more accessible by favouring the 'up' conformation of the $\mathrm{RBD}^{16}$. We conclude that the in vitro binding and neutralizing properties of CoV-X2 make it preferable over its parental antibodies.

To assess the clinical potential of CoV-X2, we investigated its ability to protect against infection and disease in an animal model. We developed a mouse model in which human ACE2 (hACE2) is expressed by cells of the upper and lower respiratory tract after inhalation of a modified adeno-associated virus (AAV) (AAV-hACE2) (Fig. 2, Extended Data Fig. 7, Methods).

This approach enables the rapid production of large cohorts of mice, and has the advantage of being applicable to wild-type and mutant mouse colonies, independently of age and sex. Moreover, because AAV vectors are only weakly immunogenic and cytotoxic, the system allows for prolonged expression of hACE2 ${ }^{17-20}$ (Extended Data Fig. 7). SARS-CoV-2 infection of ACE2-humanized mice (hereafter, hACE2 mice) results in progressive weight loss, respiratory pathology and disease that require culling at 8 days post-infection (dpi) (Fig. 2a-c, Extended Data Fig. 7).
To evaluate the protective effect of antibodies, we treated hACE2 mice one day before SARS-CoV-2 infection (150 $\mu \mathrm{g})$ (Fig. 2d, e, g, h) or $12 \mathrm{~h}$ after challenge $(250 \mu \mathrm{g})$ (Fig. $2 \mathrm{~d}, \mathrm{f})$ and monitored them over time. Upon intranasal infection with $1 \times 10^{4}$ plaque-forming units of SARS-CoV-2 (SARS-CoV-2/human/Czech Republic/951/2020), mice treated with an isotype control (an antibody against the envelope protein of Zika virus) showed weight loss starting at $3 \mathrm{dpi}$; by $8 \mathrm{dpi}$, most mice had lost approximately $25-30 \%$ of their body weight, reaching the end point for humane culling (Fig. 2e,f). We recovered infectious virus from the lungs (Fig. 2g), and detected viral RNA in the spleen (Fig. 2h) but not in the heart (data not shown). The lung pathology of these mice resembled that associated with severe COVID-19 in humans ${ }^{21}$, and was characterized by diffuse alveolar damage (50-80\% of tissue area), alveolar replacement with infiltrates of immune cells and fibroblasts, thickened septa and infiltrations by activated macrophages with foamy cytoplasm (Fig. 2j). By contrast, mice treated with CoV-X2 maintained their body weight $(P<0.0001$ at $4-8 \mathrm{dpi}$, when compared to mice treated with isotype control) (Fig. $2 \mathrm{e}$; the $P$ values between all groups are given in Extended Data Table 1), had a reduced viral burden in lungs and viral RNA levels in spleen (Fig. $2 \mathrm{~g}, \mathrm{~h}$ ) and displayed neither macro- nor histopathological changes (diffuse alveolar damage of less than 5-10\%) (Fig. 2i, j). Although infectious virus was detected at $2 \mathrm{dpi}$ in all mice that were treated with $\mathrm{C} 121(n=5), \mathrm{C} 135(n=5)$ or control $(n=8)$, in mice that received CoV-X2 it was detected only at low levels in one mouse (out of five) (Fig. $2 \mathrm{~g}$ ). At $5 \mathrm{dpi}$, we readily recovered infectious virus from control-treated mice ( 5 of 6 mice), but only from 1 out of 10 mice treated with $\mathrm{CoV}-\mathrm{X} 2$ (Fig. $2 \mathrm{~g}$ ). Furthermore, CoV-X2 was also protective when administered at $12 \mathrm{~h}$ after SARS-CoV-2 challenge (Fig. 2d,f). As none of the mice treated with CoV-X2 exhibited symptoms at any time, we conclude that $\mathrm{CoV}-\mathrm{X} 2$ protects mice from the disease.

Because monotherapy with $\mathrm{C} 121$ or $\mathrm{C} 135$ monoclonal antibodies leads to virus escape in vitro ${ }^{14}$, we treated hACE2 mice with the individual antibodies and sequenced the virus. Only wild-type RBD sequences were obtained from control mice $(n=10)$. In mice treated with C121, there was selection for a mutation that results in the E484D substitution ( 5 of 5 mice that were analysed at $8 \mathrm{dpi}$ ). Escape mutations at E484 generated by $\mathrm{C} 121$ have previously been observed in vitro ${ }^{14}$, and changes at this residue (which are also present in the B.1.351 and P.1 variants) reduce neutralization by human sera by more than tenfold ${ }^{22}$. The E484D substitution affects intermolecular hydrogen bonds at the core of the interface between $\mathrm{C} 121$ and the RBD, and it has previously been suggested to increase the affinity of the RBD for ACE ${ }^{23}$. Virus containing Asp at position 484 is pathogenic, as 7 out of 9 mice treated with $\mathrm{C} 121$ developed disease (Fig. 2e) and only this virus was found in their lungs. By contrast (and unlike the in vitro results ${ }^{14}$ ), we did not observe virus evasion or pathology in mice treated with $\mathrm{C135}(n=5)$ (Fig. 2e and data not shown). Even though we did not retrieve infectious virus (at $8 \mathrm{dpi}$ ) from mice treated with CoV-X2 $(n=13)$ and noticed no symptoms in these mice, we detected low levels of residual viral RNA in some mice after 40 cycles of PCR amplification: in 6 of 13 mice, the virus sequence was wild type, and in 2 mice the overlapping sequencing traces were consistent with the coexistence of wild-type virus and a variant with Asp at position 484. Thus, in these 2 (out of 13) mice that contained the E484D variant, $\mathrm{CoV}-\mathrm{X} 2$ remained protective even if the mutation diluted the effective antibody concentration (presumably leaving only the $\mathrm{C} 135$ moiety active).

Monoclonal antibodies that target the SARS-CoV-2S are in advanced clinical trials and show promise against COVID-19 ${ }^{1,2}$. Concomitant use of multiple antibodies is preferred for increased efficacy and adding resistance against viral evasion. Indeed, the virus can escape pressure by a single antibody in vitro and-as shown here-also in mice. Moreover, RBD mutations that threaten the efficacy of single monoclonal antibodies have already been detected in virus that is circulating in minks and humans, including mutations at the $\mathrm{C} 121$ and $\mathrm{C} 135$ epitopes (Extended Data Fig. 8). One disadvantage of antibody cocktails is the requirement 

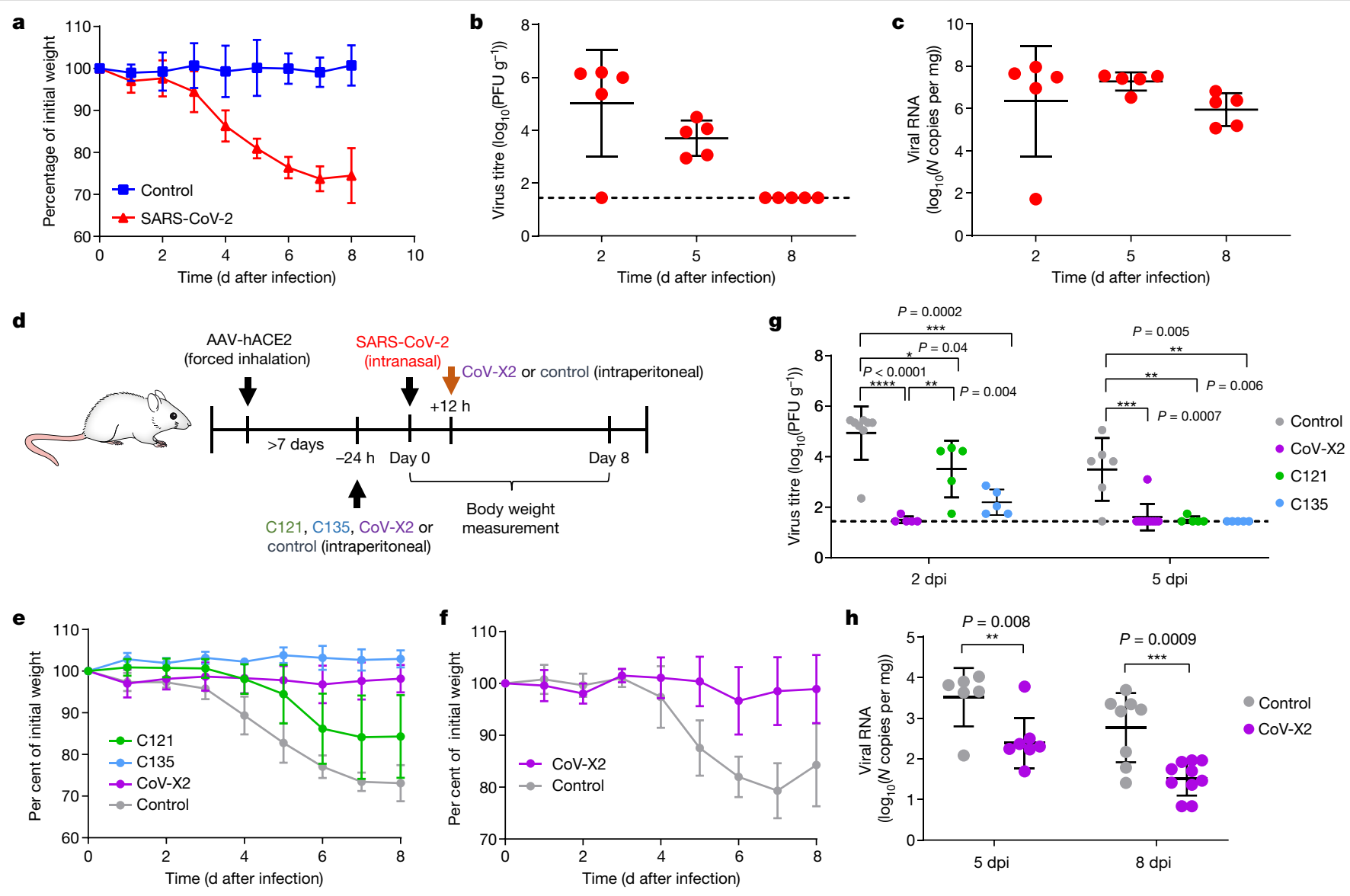

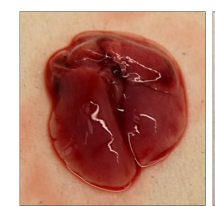

Isotype control

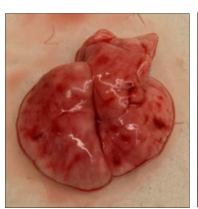

C135

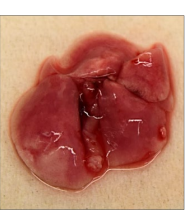

C121

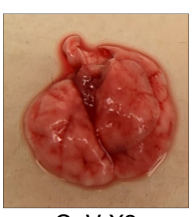

CoV-X2

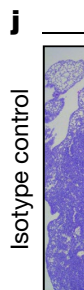

$H \& E$

$\mathrm{F} 4 / 80 \mathrm{IHC}$

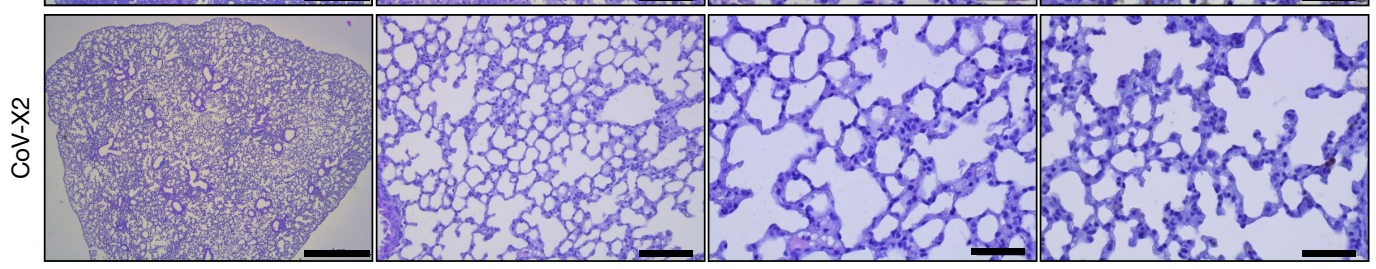

Fig. 2 | CoV-X2 protects mice transduced with AAV-hACE2 against SARS-CoV-2 disease. a, Body weight over time in mice infected with SARS-CoV-2. We transduced 13-15-week-old C57BL/6NCrl wild-type female mice with AAV-hACE2 by forced inhalation, delivering viral particles to the upper and lower respiratory tract. After $>7$ days, mice were infected with SARS-CoV- $2\left(1 \times 10^{4}\right.$ plaque-forming units $\left.(\mathrm{PFU})\right)$ or received vehicle by the intranasal route. Weight was monitored daily for 8 days (SARS-CoV-2, $n=5$; control, $n=4)$. Mean with s.d. $\mathbf{b}$, Kinetics of viral burden in lungs of mice infected with SARS-CoV-2 $(n=5)$ by plaque assays. Mean with s.d. Dashed line, limit of detection. c, Kinetics of viral RNA levels in lung samples from mice infected with SARS-CoV-2 $(n=5)$ by quantitative PCR with reverse transcription (RT-qPCR). Mean with s.d.d, Wild-type mice were transduced with AAV-hACE2 by forced inhalation. After $>7$ days, mice were inoculated intraperitoneally with antibodies one day before (black arrow) or $12 \mathrm{~h}$ after (red arrow) being infected intranasally with SARS-CoV-2 $\left(1 \times 10^{4} \mathrm{PFU}\right)$. e, f, Body weight was monitored daily in mice treated $24 \mathrm{~h}$ before infection (e, C121, $n=9 ; \mathrm{C} 135, n=5 ; \mathrm{CoV}-\mathrm{X} 2$, $n=13$; isotype control, $n=10$ ) or $12 \mathrm{~h}$ after infection (f, $\mathrm{CoV}-\mathrm{X} 2, n=4$; control, $n=10$ ). Mean with s.d. is shown. $g$, Lung viral burden by plaque assay at $2 \mathrm{dpi}$ (isotype control, $n=8 ; \mathrm{CoV}-\mathrm{X} 2, n=5 ; \mathrm{C} 121, n=5 ; \mathrm{C} 135, n=5$ ) and $5 \mathrm{dpi}$ (isotype control, $n=6 ; \mathrm{CoV}-\mathrm{X} 2, n=10 ; \mathrm{C} 121, n=5 ; \mathrm{C} 135, n=5)$. The dashed line indicates the limit of detection; mean with s.d.Pvalue was calculated with two-tailed Student's $t$-test. h, Viral RNA levels in the spleen by RT-qPCR at 5 and $8 \mathrm{dpi}$ (isotype control, $n=6$ or 8 (indicated by dots); CoV-X2, $n=8$ or 10 (indicated by dots)). Mean with s.d. $P$ value, two-tailed Student's $t$-test.i, Photographs of lungs from infected mice ( $8 \mathrm{dpi}) . \mathbf{j}$, Histopathology and F4/80 immunohistochemistry (IHC). Haematoxylin and eosin (H\&E)-stained sections of paraffin-embedded lungs from infected mice ( $8 \mathrm{dpi})$. Arrowheads, foamy macrophages. F4/80 IHC shows abundant macrophage infiltration in lungs of mice treated with isotype control, but not those with $\mathrm{CoV}-\mathrm{X} 2$. Each image representative of two separate experiments ( $n=3-5$ mice per group). Scale bars, $20 \mu \mathrm{m}$ (inset in H\&E), $50 \mu \mathrm{m}$ (H\&E right, F4/80 IHC), $100 \mu \mathrm{m}$ (H\&E middle), $1 \mathrm{~mm}(\mathrm{H} \& \mathrm{E}$, left). 
for a development and production capacity twice or more that required for single monoclonal antibodies, which is a considerable challenge in light of the increased demand owing to COVID-19 related vaccines and therapeutic agents (on top of the need to maintain production of biologics for other diseases $)^{4}$.

Multispecific antibodies offer the advantages of cocktails in a single molecule. We have shown that the CoV-X2 bispecific antibody is more effective than the related monoclonal antibodies at inhibiting ACE2 binding; it has a sub-nanomolar $\mathrm{IC}_{50}$ against a broader array of viral sequences and it protect mice from SARS-CoV-2 even when $\mathrm{C} 121$ (its potent parental monoclonal antibody) fails owing to viral escape. $\mathrm{C} 135$, the other parental monoclonal antibody, did not generate escape mutants in our mouse experiment, but readily generated them in vitro ${ }^{14}$. $\mathrm{CoV}-\mathrm{X} 2$ is expected to be more resistant to viral escape as compared to monoclonal antibodies. We have shown that $\mathrm{CoV}-\mathrm{X} 2$ binds and neutralizes mutants that are not recognized by its parental monoclonal antibodies, as well as variants of concern that have recently been detected in the UK ${ }^{12}$, South Africa ${ }^{13}$ and Brazil ${ }^{24}$.

Unlike other multispecific antibodies ${ }^{25}, \mathrm{CoV}-\mathrm{X} 2$ is a fully human IgG-like molecule. As such, it is suitable for further development and could be engineered to alter effector functions. For example, the Fragment crystallizable (Fc) of CoV-X2 has already been modified by introducing LALA-PG mutations ${ }^{26}$ to modulate its interaction with Fc receptor and complement without affecting its antigen-binding properties. The LALA modification prevents antibody-dependent enhancement of flavivirus infection ${ }^{27}$ and may be a desirable modification in the context of SARS-CoV-2, as cellular and animal experiments with coronaviruses (including SARS-CoV ${ }^{28,29}$ ) support the possibility of antibody-dependent enhancement. Other modifications (such as the LS modification ${ }^{26}$ for increased half-life) are easily achievable. Finally, CoV-X2 is human-derived and produced in the CrossMab format, which has already shown to be safe in clinical trials ${ }^{30}$, which further supports the possibility of future development. IgG-like bispecific antibodies are therefore worth adding to the tools that we use to address SARS-CoV-2 and its future mutations.

\section{Online content}

Any methods, additional references, Nature Research reporting summaries, source data, extended data, supplementary information, acknowledgements, peer review information; details of author contributions and competing interests; and statements of data and code availability are available at https://doi.org/10.1038/s41586-021-03461-y.

1. DeFrancesco, L. COVID-19 antibodies on trial. Nat. Biotechnol. 38, 1242-1252 (2020).

2. Klasse, P. J. \& Moore, J. P. Antibodies to SARS-CoV-2 and their potential for therapeutic passive immunization. eLife $\mathbf{9}$, e57877 (2020).
3. Robbiani, D. F. et al. Convergent antibody responses to SARS-CoV-2 in convalescent individuals. Nature 584, 437-442 (2020).

4. Ecker, D. M. \& Seymour, P. in CPhl Annual Report 2020: Postulating the Post-COVID Pharma Paradigm, 43-49 (Informamarkets, 2020).

5. Baum, A. et al. REGN-COV2 antibodies prevent and treat SARS-CoV-2 infection in rhesus macaques and hamsters. Science 370, 1110-1115 (2020).

6. Schäfer, A. et al. Antibody potency, effector function, and combinations in protection and therapy for SARS-CoV-2 infection in vivo. J. Exp. Med. 218, e20201993 (2021).

7. Schlake, T. et al. mRNA: a novel avenue to antibody therapy? Mol. Ther. 27, 773-784 (2019).

8. Tiwari, P. M. et al. Engineered mRNA-expressed antibodies prevent respiratory syncytial virus infection. Nat. Commun. 9, 3999 (2018).

9. Barnes, C. O. et al. SARS-CoV-2 neutralizing antibody structures inform therapeutic strategies. Nature 588, 682-687 (2020).

10. Schaefer, W. et al. Immunoglobulin domain crossover as a generic approach for the production of bispecific IgG antibodies. Proc. Natl Acad. Sci. USA 108, 11187-11192 (2011).

11. Walls, A. C. et al. Structure, function, and antigenicity of the SARS-CoV-2 spike glycoprotein. Cell 181, 281-292.e6 (2020).

12. Kemp, S. et al. Recurrent emergence and transmission of a SARS-CoV-2 spike deletion $\Delta$ H69/V7O. Preprint at https://doi.org/10.1101/2020.12.14.422555 (2020).

13. Tegally, H. et al. Emergence and rapid spread of a new severe acute respiratory syndrome-related coronavirus 2 (SARS-CoV-2) lineage with multiple spike mutations in South Africa. Preprint at https://doi.org/10.1101/2020.12.21.20248640 (2020).

14. Weisblum, Y. et al. Escape from neutralizing antibodies by SARS-CoV-2 spike protein variants. eLife 9 , e61312 (2020).

15. Schmidt, F. et al. Measuring SARS-CoV-2 neutralizing antibody activity using pseudotyped and chimeric viruses. J. Exp. Med. 217, e2O201181 (2020).

16. Benton, D. J. et al. The effect of the D614G substitution on the structure of the spike glycoprotein of SARS-CoV-2. Proc. Natl Acad. Sci. USA 118, e2O22586118 (2021)

17. Han, K. et al. Lung expression of human ACE2 sensitizes the mouse to SARS-CoV-2 infection. Am. J. Respir. Cell Mol. Biol. 64, 79-88 (2021).

18. Hassan, A. O. et al. A SARS-CoV-2 infection model in mice demonstrates protection by neutralizing antibodies. Cell 182, 744-753.e4 (2020).

19. Sun, J. et al. Generation of a broadly useful model for COVID-19 pathogenesis, vaccination, and treatment. Cell 182, 734-743.e5 (2020).

20. Sun, S. H. et al. A mouse model of SARS-CoV-2 infection and pathogenesis. Cell Host Microbe 28, 124-133.e4 (2020).

21. Deshmukh, V., Motwani, R., Kumar, A., Kumari, C. \& Raza, K. Histopathological observations in COVID-19: a systematic review. J. Clin. Pathol. 74, 76-83 (2021).

22. Greaney, A. J. et al. Comprehensive mapping of mutations to the SARS-CoV-2 receptor-binding domain that affect recognition by polyclonal human serum antibodies. Cell Host Microbe 29, 463-476 (2021).

23. Chen, J., Wang, R., Wang, M. \& Wei, G. W. Mutations strengthened SARS-CoV-2 infectivity. J. Mol. Biol. 432, 5212-5226 (2020).

24. Sabino, E. C. et al. Resurgence of COVID-19 in Manaus, Brazil, despite high seroprevalence. Lancet 397, 452-455 (2021).

25. Dong, J. et al. Development of humanized tri-specific nanobodies with potent neutralization for SARS-CoV-2. Sci. Rep. 10, 17806 (2020).

26. Saunders, K. O. Conceptual approaches to modulating antibody effector functions and circulation half-life. Front. Immunol. 10, 1296 (2019).

27. Dejnirattisai, W. et al. Cross-reacting antibodies enhance dengue virus infection in humans. Science 328, 745-748 (2010).

28. Yip, M. S. et al. Antibody-dependent infection of human macrophages by severe acute respiratory syndrome coronavirus. Virol. J. 11, 82 (2014).

29. Yip, M. S. et al. Antibody-dependent enhancement of SARS coronavirus infection and its role in the pathogenesis of SARS. Hong Kong Med. J. 22 (Suppl 4), 25-31 (2016).

30. Klein, C. et al. Engineering therapeutic bispecific antibodies using CrossMab technology. Methods 154, 21-31 (2019).

Publisher's note Springer Nature remains neutral with regard to jurisdictional claims in published maps and institutional affiliations.

(c) The Author(s), under exclusive licence to Springer Nature Limited 2021 


\section{Methods}

No statistical methods were used to predetermine sample size. The experiments were not randomized, and investigators were not blinded to allocation during experiments and outcome assessment.

\section{Protein expression and purification}

To express SARS-CoV-2 S, a codon-optimized gene encoding residues 1-1208 (GenBank (https://www.ncbi.nlm.nih.gov/genbank/): MN908947) with proline substitutions at residues 986 and 987, a 'GSAS' substitution at the furin cleavage site (residues 682-685), a C-terminal T4 fibritin trimerization motif and a C-terminal $8 \times$ HisTag was synthesized and cloned into the mammalian expression vector pcDNA3.1(+) by Genscript. Codon-optimized nucleotide sequences encoding SARS-CoV-2 RBD (residues 319-591; GenBank: MN908947) with a C-terminal $8 \times$ His Tag was obtained from Genscript. hACE2 fused to the $\mathrm{Fc}$ region of mouse IgG at the $\mathrm{C}$ terminus was also synthesized and cloned into the mammalian expression vector pcDNA3.1(+). All proteins were produced by transient PEI transfection in Expi293F cells (ThermoFisher), purified from the cell supernatants 6 days after transfection by HiTrap Chelating HP (Cytiva) or HiTrap Protein A HP (Cytiva), analysed by SDS-PAGE and dynamic light scattering on a DynaPro NanoStar (Wyatt Technology, software Dynamics v.7.1.7.16). RBD and S trimer mutations were introduced in the plasmids by gene synthesis (Genscript) and purified. All proteins underwent quality control and biophysical characterization to ensure functionality, stability, lack of aggregation and batch-to-batch reproducibility.

Monoclonal antibody production. Plasmids containing the coding sequence for the production of the monoclonal antibodies $\mathrm{C} 121, \mathrm{C} 135$, and C144 were prepared as previously described ${ }^{3}$. These monoclonal antibodies were produced by transient PEI transfection in Expi293F cells (ThermoFisher), purified from the cell supernatants 6 days after transfection by HiTrap Protein A HP (Cytiva) and HiLoad Superdex 200 16/60 column (Cytiva).

Design, expression and purification of bispecific antibodies. Bispecific antibodies in the single-chain Fv format (CoV-scB1 and CoV-scB2) were designed from the sequences of the variable regions of monoclonal antibodies $\mathrm{C} 144$ and $\mathrm{C} 135$ (CoV-scB1) or $\mathrm{C} 121$ and $\mathrm{C} 135^{3}$ (CoV-scB2), following a previously described method ${ }^{31}, \mathrm{~N}$-terminal signal peptides (residues 1-19; UniProt (https://www.uniprot.org/): P01743) and a C-terminal $6 \times$ HisTag were added. The constructs were synthesized and subcloned into the mammalian expression vector pcDNA3.1(+) by Genscript. The single-chain bispecific antibodies were produced by transient PEI transfection in Expi293F cells (ThermoFisher), purified from the cell supernatants 6 days after transfection by HiTrap Chelating HP (Cytiva) and HiLoad Superdex $7516 / 60$ column (Cytiva).

Bispecific antibodies in the CrossMAb format were designed from the sequences of the variable regions of antibodies C144 and C135 (CoV-X1) or $\mathrm{C} 121$ and $\mathrm{C}_{135}{ }^{3}$ (CoV-X2). Light and heavy chain constant region sequences (UniProt P01834 and UniProt P01857) were added. The CrossMAbs were designed, as previously described ${ }^{10}$, with $\mathrm{CH} 1-\mathrm{CL}$ crossover in the $\mathrm{C} 135$ moiety. Four constructs, one for each light chain ( $\mathrm{LC}(\mathrm{C} 144), \mathrm{LC}(\mathrm{C121})$ and $\left.\mathrm{LC}^{\mathrm{CH1}-\mathrm{CL}}(\mathrm{C} 135)\right)$ and heavy chain $\left(\mathrm{HC}(\mathrm{C} 144), \mathrm{HC}(\mathrm{C121})\right.$ and $\left.\mathrm{HC}^{\mathrm{CH1}-\mathrm{Cl}}(\mathrm{C} 135)\right)$ were synthesized and subcloned into the mammalian expression vector pcDNA3.1(+) by Genscript. Signal peptides were included at the $N$ terminus of the variable sequences for expression purposes (residues 1-19; UniProt P01743 for the heavy chains and residues 1-20; UniProt P06312 for the light chains). The antibodies were produced by PEI transient transfection, plasmid ratio 1:1:1:1, in Expi293F cells (ThermoFisher), purified from the cell supernatants 6 days after transfection by HiTrap Protein A HP (Cytiva), and HiLoad Superdex 200 16/60 column (Cytiva).
All antibodies underwent quality control and biophysical characterization to ensure functionality, stability, lack of aggregation and batch-to-batch reproducibility.

\section{Computational modelling}

$\mathrm{CoV}$-scB1 and $\mathrm{CoV}$-scB2 were modelled starting from the variable fragment of available experimental structures of the parental antibodies (Protein Data Bank (PDB) (https://www.rcsb.org/) identifiers: 7K8X for $\mathrm{C} 121,7 \mathrm{~K} 8 \mathrm{Z}$ for $\mathrm{C} 135$ and 7K90 for $\mathrm{C144}$ ); the connecting linkers were manually added (Pymol); the stability and feasibility of the bispecific constructs on the $S$ trimer were manually and visually investigated according to structural biology considerations.

$\mathrm{CoV}-\mathrm{X} 1$ and $\mathrm{CoV}-\mathrm{X} 2$ were assembled starting from the experimental structures of individual Fab antibodies in complex with SARS-CoV-2 (PDB 7K8X for C121, 7K8Z for C135 and 7K90 for C144). The Fc moiety was manually placed in proximity to the $C$ terminus of each Fab heavy chain; $\mathrm{CH} 1$ domains were then connected to the Fc using the ALMOST toolkit ${ }^{32}$, thus obtaining the full antibody structures.

The S trimer coordinates were obtained from available experimental structures; loop regions not present in the structure were modelled using the I-TASSER suite ${ }^{33}$. PDB 6VXX served as a basis for the 'three down' conformation; PDB 6VYB for the 'one RBD up' conformation; and PDB 7A93 for the 'two RBD up' conformation. Conformations not directly available (for example, trimer with 'three RBD up') were obtained by structural alignment and repetition of the appropriate conformation in the $\mathrm{S}$ monomer structures (for example, monomeric 'RBD up') using the PyMol software suite ${ }^{34}$

All the combinations of S trimer (three RBD down, one up, two up and three up) and antibodies (bispecific antibodies formed by combinations of C121, C135 and C144) were subjected to 400 ns or 200 ns of fully atomistic molecular dynamics simulations to obtain energetically favourable and stable conformations using GROMACS ${ }^{35}$. Calculations were performed on the CINECA-Marconi100 supercomputer.

The system was initially set up and equilibrated through standard molecular dynamics protocols: proteins were centred in a triclinic box, $0.2 \mathrm{~nm}$ from the edge, filled with SPCE water model and $0.15 \mathrm{M}$ $\mathrm{Na}^{+} \mathrm{Cl}^{-}$ions using the AMBER99SB-ILDN protein force field. Energy minimization was performed to let the ions achieve a stable conformation. Temperature and pressure equilibration steps, respectively, at $310 \mathrm{~K}$ and 1 bar of 100 ps each were completed before performing the full molecular dynamics simulations with the above-mentioned force field. Molecular dynamics trajectory files were analysed after removal of periodic boundary conditions. The stability of each simulated complex was verified by root mean square deviation and visual analysis.

\section{SPR}

The antibody binding properties were analysed at $25^{\circ} \mathrm{C}$ on a Biacore $8 \mathrm{~K}$ instrument (GE Healthcare) using $10 \mathrm{mM}$ HEPES pH 7.4, $150 \mathrm{mM} \mathrm{NaCl}$, 3 mM EDTA and $0.005 \%$ Tween-20 as running buffer.

SARS-CoV-2 RBD or full S, and their mutants, were immobilized on the surface of CM5 chips (Cytiva) through standard amine coupling. Increasing concentrations of antibody $(3.12,6.25,12.5,25$ and $50 \mathrm{nM})$ were injected using a single-cycle kinetics setting; analyte responses were corrected for unspecific binding and buffer responses. Curve fitting and data analysis were performed with Biacore Insight Evaluation Software v.2.0.15.12933.

Competition experiments were performed to assess the ability of $\mathrm{COV}-\mathrm{X} 2$ to bind its target with both arms. A low amount of RBD $(5 \mathrm{nM})$ was immobilized on the surface of a CM5 chip through standard amine coupling. $\mathrm{C} 121$ or $\mathrm{C} 135$ antibodies were injected at a high concentration $(1.5 \mu \mathrm{M})$ to saturate the corresponding binding sites on the RBD; CoV-X2 $(200 \mathrm{nM})$ was subsequently injected. The same experimental setting was performed with a different injection order as a control: COV-X2 $(1.5 \mu \mathrm{M})$ injected to saturate RBD binding sites and subsequent injection of C121 or C135 (200 nM). 
Analysis and comparison of kinetics parameters at different RBD concentrations were performed as following. RBD was immobilized on the surface of a CM5 chip at 5, 15, 75 and $150 \mathrm{nM}$. Increasing concentrations of antibody $(3.12,6.25,12.5,25$ and $50 \mathrm{nM})$ were injected using a single-cycle kinetics setting; analyte responses were corrected for unspecific binding and buffer responses. Curve fitting and data analysis were performed with Biacore Insight Evaluation Software v.2.0.15.12933.

\section{Binding inhibition of hACE2}

ELISAs were used to investigate the ability of antibodies to inhibit the binding of S to hACE2. Each experiment was performed in duplicate, reporting the mean of the two replicates; error bars represent the s.d. of the measured values. We used 96-well ELISA plates, coated at $4{ }^{\circ} \mathrm{C}$ with $37 \mathrm{nMS}$, and washed and blocked with PBS $+10 \%$ FCS. Antibodies were then added either at constant saturating concentration $(160 \mathrm{nM})$ or at different dilutions (starting from $340 \mathrm{nM}$ and serially diluted 1 to 3) and incubated $1 \mathrm{~h}$ at $25^{\circ} \mathrm{C}$; after washing, hACE2-mouse Fc was added either at constant saturating concentration $(160 \mathrm{nM})$ or at different dilutions (starting from $340 \mathrm{nM}$ and serially diluted 1 to 2 ) and left $1 \mathrm{~h}$ at $25^{\circ} \mathrm{C}$. After further washing, bound hACE2 was detected using standard protocols with goat anti-mouse IgG coupled to alkaline phosphatase (dilution 1:500, SouthernBiotech). ELISA plates were measured with the reader software Gen5 version 1.11.5 (BioTek Instruments). Data were analysed with Microsoft Excel 2016 and GraphPad Prism version 8.4.2.

\section{SARS-CoV-2 pseudotyped reporter virus and pseudotyped-virus} neutralization assay

A panel of plasmids expressing RBD-mutant SARS-CoV-2 $\mathrm{S}$ in the context of pSARS-CoV-2- $\mathrm{S}_{\Delta 19}$ have previously been described ${ }^{14,36}$. The mutant 'KEN' (K417N/E484K/N501Y) was constructed in the context of a pSARS-CoV-2-S $\mathrm{S}_{\Delta 19}$ variant with a mutation in the furin cleavage site (R683G). The IC ${ }_{50}$ of these pseudotypes were compared to a wild-type SARS-CoV-2 S sequence carrying R683G. Generation of SARS-CoV-2 pseudotyped HIV-1 particles was performed as previously described ${ }^{3,15}$.

The neutralization activity of the bispecific and monoclonal antibodies was measured as previously reported ${ }^{3,15}$. In brief, fourfold serially diluted antibodies were incubated with SARS-CoV-2 pseudotyped virus for $1 \mathrm{~h}$ at $37^{\circ} \mathrm{C}$. The mixture was subsequently incubated with 293T cells expressing ACE2 for $48 \mathrm{~h}$; the cells were washed twice with PBS and lysed with Luciferase Cell Culture Lysis $5 \times$ reagent (Promega). NanoLuc activity in lysates was measured using the Nano-Glo Luciferase Assay System (Promega) with the Modulus II Microplate Reader User interface (TURNER BioSystems). The obtained relative luminescence units (which are indicative of virus presence) were normalized to those derived from cells infected with SARS-CoV-2 pseudotyped virus in the absence of antibodies. The $\mathrm{IC}_{50}$ was determined using four-parameter nonlinear regression (GraphPad Prism).

\section{SARS-CoV-2 virus-neutralization assay}

The neutralizing activity of CoV-X2 against SARS-CoV-2 wild type, B.1 (D614G), B.1.1.7, P.1 and B.1.351 was investigated by plaque-reduction neutralization tests following a previously reported protocol ${ }^{37}$. In brief, $50 \mu \mathrm{l}$ of antibody, starting from a concentration of $12 \mu \mathrm{g} \mathrm{ml}^{-1}$ or $190 \mu \mathrm{g} \mathrm{ml}^{-1}$ in a serial threefold dilution, were mixed in a flat-bottomed tissue culture microtitre plate (COSTAR) with an equal volume of 100 median tissue culture infectious dose of infectious virus that was isolated from patients with COVID-19, sequenced, titrated and incubated at $33{ }^{\circ} \mathrm{C}$ in $5 \% \mathrm{CO}_{2}$. After $1 \mathrm{~h}, 3 \times 10^{4}(100 \mu \mathrm{l})$ Vero E6 cells (VERO C1008, Vero 76, clone 18 E6, Vero E6; ATCC CRL-1586) were added to each well. After 3 days of incubation, cells were stained with Gram's crystal violet solution (Merck) plus 5\% formaldehyde $40 \% \mathrm{~m} / \mathrm{v}$ (Carlo Erba S.p.A.) for $30 \mathrm{~min}$. Microtitre plates were then washed in water. Wells were analysed to evaluate the degree of cytopathic effect compared to untreated control. Each experiment was performed in triplicate.
The $\mathrm{IC}_{50}$ was determined using three-parameter nonlinear regression (GraphPad Prism).

\section{AAV-hACE2 vector design}

Plasmid design and construction. The AAV transfer plasmid expressing hACE2 (AAV-hACE2) was created by replacing the GFP sequence with ACE2 cDNA obtained from the HEK293 cell line (no. CRL-1573, ATCC, mycoplasma-free, population doubling lower than 13) in a pAAV-GFP plasmid (no. AAV-400, Cellbiolabs). HEK293 cells were cultured in Dulbecco's modified Eagle's medium (DMEM) (no. D5796, Sigma Aldrich) supplemented with $10 \%$ FBS (no. 10082139, Gibco) and $1 \%$ penicillinstreptomycin (no. XC-A4122100, BioSera). Cells were kept at $37^{\circ} \mathrm{C}$ under $5 \% \mathrm{CO}_{2}$ atmosphere and $95 \%$ humidity.

To obtain the hACE2 sequence, total RNA was isolated from a confluent $10-\mathrm{cm}^{2}$ plate of HEK293 cells using RNeasy Mini Kit (Qiagen, no. 74104) according to the manufacturer's protocol and reverse-transcribed with M-MLV Reverse Transcriptase (Promega, no. M1701). The generated cDNA was used as a template for PCR amplification with a pair of primers ( $A C E 2$ forward: 5'ATGTCAAGCTCTTCCTGG 3', ACE2 reverse: 5'CTAAAAGGA GGTCTGAACATC 3') specific for hACE2 (NM_021804.3) using Phusion High-Fidelity DNA Polymerase (NEB, no. M0530S). The PCR product was separated in a 1\% agarose gel (SeaKem LE AGAROSE; East Port, no. 50004); the band of appropriate size (2,418 bp) was extracted using NucleoSpin Gel and PCR Clean-up (Takara, no. 740609) according to the manufacturer protocol. The extracted product was treated with DreamTaq Green DNA Polymerase (Thermo Scientific, no. EP0711) in the presence of dATP to add 3'A overhangs to the PCR product. The product was then subcloned into pGEM-T Easy Vector (Promega, no. A1360). Proper insertion of the product was assessed by HindIII (Thermo Scientific, no. ER0501) and Sacl (Thermo Scientific, no. ER1132) double-digestion control. Kozak sequence and Spel recognition site were added at the $5^{\prime}$ and 3 ' end of the ACE2cDNA PCR product, respectively, by amplification with a specific pair of primers (hACE2_Kozak_Fw:5'-CAGGGGACGATGTCAAGCTCTTCCTGG -3', hACE2_Spe_Rv: 5'-ACTAGTGATCTAAAAGGAGGT-3') using Phusion High-Fidelity DNA Polymerase (NEB, no. M0530S). The amplified product was separated in $1 \%$ agarose gel (SeaKem LE AGAROSE; East Port, no. 50004), extracted with NucleoSpin Gel and PCR Clean-up (Takara, no. 740609) according to the manufacturer's protocol. The extracted ACE2 sequence was PCR-amplified with a pair of primers with microhomology arms (hACE2_IF_Fw:5'-TTCGAACATCGATTGCAGGGGACGATGTCAAG-3', hACE2_Spe_IF_Rv:5'-GCGCTGCTCGAGGCAACTAGTGATCTAAAAGGAGGT $\left.-3^{\prime}\right)$ and Phusion High-Fidelity DNA Polymerase (NEB, no. M0530S), and was subsequently purified from agarose gel with NucleoSpin Gel and PCR Clean-up (Takara, no. 740609).

The GFP sequence in the pAAV-GFP control vector (no. AAV-400, Cellbiolabs) was excised by double digestion using EcoRI (Thermo Scientific, no. ER0271)/HindIII (Thermo Scientific, no. ER0501) restriction enzymes and replaced with the hACE2 sequence flanked by microhomology arms using the In-Fusion cloning system (In-Fusion HD Cloning Kit, Takara Bio Europe, no. 639648) according to the manufacturer's protocol. Proper insertion and presence of the Spel recognition site were confirmed by double digestion using HindIII (Thermo Scientific, no. ER0501)/Mlul (ThermoScientific, no.ER0562) and Spel (Thermo Scientific, no. ER1252), respectively. The generated AAV-hACE2 vector was sequenced (Eurofins Genomics) using the following primers: CMV_Fw: 5'-AAATGGGCGGTAGGCGTG-3', seq_hACE2_start1: 5'-TGGAGATCTGAGGTCGG-3', seq_hACE2_start2: 5'-TCTTCCTCCCACAGCTCCT-3', seq_hACE2_1: 5'-CAGTTGATT GAAGATGTGGA-3', seq_hACE2_2: 5'-AGAAGTGGAGGTGGATG-3', seq_hACE2_3:5'-AGAACTGAAGTTGAAAAGG-3'. The produced hACE2 sequence was $100 \%$ identical to the reference hACE2 sequence (NM_021804.3).

Transfection of Neuro-2a cells. Neuro-2a cells (CCL-131, ATCC, mycoplasma-free, population doubling lower than 10) used for 
validation of AAV-hACE2 function were cultured in DMEM (no. D5796, Sigma Aldrich) supplemented with 10\% FBS (no. 10082139, Gibco) and $1 \%$ penicillin-streptomycin (no. XC-A4122100, BioSera) and kept at $37^{\circ} \mathrm{C}$ under $5 \% \mathrm{CO}_{2}$ atmosphere and $95 \%$ humidity. Three $60-\mathrm{mm}^{2}$ plates were seeded each with $4 \times 10^{5}$ cells and transfected the next day with the AAV-hACE2 vector using Lipofectamine 2000 Transfection Reagent (Invitrogen, no.11668027) according to the manufacturer's protocol for the 6-well plate transfection. Transfection with pAAV-GFP control vector (no. AAV-400, Cellbiolabs) and non-transfected HEK293 cells were used as negative controls. After $48 \mathrm{~h}$, cells were collected for western blotting in RIPA buffer, supplemented with AEBSF protease inhibitor (AppliChem, no. A1421) and cOmplete Mini Protease Inhibitor Cocktail (Sigma Aldrich, no. 04693124001). Expression of hACE2 in Neuro-2a cells was compared to the non-transfected HEK293 cells.

\section{AAV-hACE2 particle production}

AAV293 cell transfection. The AAV293 cell line (Cellbiolabs, no. AAV100, mycoplasma-free, population doubling lower than 8) used for AAV production was cultured in DMEM (no. D5796, Sigma Aldrich) supplemented with $10 \%$ FBS (no.10082139, Gibco), 1\% penicillin-streptomycin (no. XC-A4122100, BioSera) and 1\% NEAA (no. M7145, Sigma Aldrich). Cells were kept at $37^{\circ} \mathrm{C}$ under $5 \% \mathrm{CO}_{2}$ atmosphere and $95 \%$ humidity. A day before transfection, $6.5 \times 10^{6}$ cells were seeded on $15-\mathrm{cm}^{2}$ cultivation plates to reach $80-90 \%$ confluency on the day of transfection. Vectors pHelper (no.340202, Cell BioLabs), AAV Rep/Cap 2/9n (no. 112865, Addgene) and AAV-hACE2 were used for transfection in equimolar ratio. The total amount of DNA ( $28 \mu \mathrm{g}$ per plate) diluted in $1 \mathrm{ml}$ per plate of DMEM was mixed with linear polyethylenimine hydrochloride, M.W. 40000 PEI (no. 24765-1, Polysciences) in 1:2.7 ratio. After 20 min of incubation at room temperature, the transfection mixture was added to a cultivation plate with FBS-reduced medium (DMEM supplemented with $1 \% \mathrm{FBS}$ ) in a dropwise manner. After $5 \mathrm{~h}$ of incubation at $37^{\circ} \mathrm{C}$ under $5 \% \mathrm{CO}_{2}$ atmosphere, the medium was removed and replaced with fresh complete growth medium (DMEM supplemented with $10 \%$ FBS and $1 \%$ penicillin-streptomycin).

AAV293-hACE2 collection. Three days after transfection, both cell medium and cells were collected for AAV particle isolation. These procedures were adapted and modified from a previous publication ${ }^{38}$. The medium was collected into 50-ml centrifuge tubes and cells were washed twice with $5 \mathrm{ml}$ of PBS. Subsequently, cells were scraped in $1 \mathrm{ml}$ of PBS and centrifuged at $1,000 \mathrm{~g}$ for $10 \mathrm{~min}$ at $4{ }^{\circ} \mathrm{C}$. The supernatant was added to the previously collected cell medium; the cell pellet was kept on ice during subsequent processing. The medium was centrifuged at $3,200 \mathrm{~g}$ for $15 \mathrm{~min}$ at $4{ }^{\circ} \mathrm{C}$ and the supernatant was then filtered into a sterile glass bottle using 0.22- $\mu \mathrm{m}$ PES membranes. PEG-8000 (no. V3011, Promega) was added to the medium in a glass bottle in a 1:4 ratio. The mixture was stirred slowly at $4{ }^{\circ} \mathrm{C}$ for $1 \mathrm{~h}$ and then incubated overnight at $4{ }^{\circ} \mathrm{C}$ without stirring to allow full virus precipitation. The following day, the medium was centrifuged at 2,800 $g$ for $20 \mathrm{~min}$ at $4{ }^{\circ} \mathrm{C}$ and the pellet resuspended in $10 \mathrm{ml}$ of PBS solution with $0.001 \%$ Pluronic F-68 non-ionic surfactant (no. 24040032, Gibco) and $200 \mathrm{mM}$ $\mathrm{NaCl}$ (no. S5886, Sigma Aldrich) and sonicated at 50\% amplitude with $4 \times 1$-s on $/ 15$-min off pulses on ice. The cell lysate was centrifuged at $3,200 \mathrm{~g}$ for $15 \mathrm{~min}$ at $4{ }^{\circ} \mathrm{C}$. Subsequently, $50 \mathrm{U} \mathrm{ml}^{-1}$ of benzonase nuclease (no. E1014-25KU, Sigma Aldrich) was added to the viral suspension to degrade any residual DNA. After incubation for $1 \mathrm{~h}$ at $37^{\circ} \mathrm{C}$, the viral suspension was centrifuged at $2,400 \mathrm{~g}$ for $10 \mathrm{~min}$ at $4^{\circ} \mathrm{C}$ and the clarified supernatant was further purified.

AAV-hACE2 purification by iodixanol gradient ultracentrifugation. A gradient consisting of $15 \%$ iodixanol (in $1 \mathrm{M} \mathrm{NaCl}, 2.7 \mathrm{mM} \mathrm{MgCl}_{2} 2 \mathrm{mM}$ $\mathrm{KCl}$ in phosphate buffer), $25 \%$ iodixanol (in $2.7 \mathrm{mM} \mathrm{MgCl}_{2}, 2 \mathrm{mM} \mathrm{KCl}$, and $0.001 \%$ phenol red in PBS), $40 \%$ iodixanol (in $2.7 \mathrm{mM} \mathrm{MgCl}_{2}$ and $2 \mathrm{mM} \mathrm{KCl}$ in PBS) and 0,002\% phenol red (no. P3532, Sigma Aldrich) in
60\% iodixanol (OptiPrep Density Gradient Medium, no. D1556, Sigma Aldrich) was prepared in QuickSeal tubes according a previous publication $^{38}$. Five $\mathrm{ml}$ of clarified viral supernatant was carefully added on the top of the gradient and the rest of the tube was filled up with PBS. Ultracentrifugation was carried out at 350,000 $\mathrm{g}$ for $90 \mathrm{~min}$ in a pre-cooled T70i rotor at $10^{\circ} \mathrm{C}$. After ultracentrifugation, approximately 750 - $\mu$ l fractions were collected from the $40 \%$ iodixanol phase using an $18 \mathrm{G}$ needle puncturing the QuickSeal tube at the interface of the $60 \%$ and $40 \%$ iodixanol.

AAV-hACE2 purity validation and buffer exchange. The purity of the collected fractions from $40 \%$ iodixanol containing AAV-hACE2 particles was assessed by SDS-PAGE. Ten $\mu$ l of each collected fraction was mixed with $3.5 \mu$ l of $4 \times$ Laemmli buffer and loaded to $4-20 \%$ Mini-PROTEAN TGX Precast Protein Gel (no. 4561096, Bio-Rad). The gels were briefly washed in $\mathrm{dH}_{2} \mathrm{O}$ and stained with silver according to the manufacturer's protocol (Pierce Silver Stain Kit, no. 10096113, Thermo Scientific).

Selected AAV-hACE2 fractions were pooled and concentrated using Amicon Ultra-0.5 Centrifugal Filter Unit (molecular weight cut-off of $100 \mathrm{kDa}$ ). First, Amicon filter membranes were activated by incubation with $0.1 \%$ pluronic $\mathrm{F}-68,0.01 \%$ pluronic $\mathrm{F}-68$ and $200 \mathrm{mM} \mathrm{NaCl}$, followed by $0.001 \%$ of pluronic F- 68 in PBS and centrifugation at $1,900 \mathrm{~g}$ for 5 min at $4{ }^{\circ} \mathrm{C}$. Pooled fractions with AAV-hACE2 particles were loaded onto activated Amicon filter membranes and centrifuged at 2,600 $g$ for $5 \mathrm{~min}$ at $4{ }^{\circ} \mathrm{C}$. The membranes were washed several times with $0.001 \%$ pluronic F-68 in PBS (centrifugation at 2,600 $\mathrm{g}$ for 8 min at $4{ }^{\circ} \mathrm{C}$ ) until the residual iodixanol was completely removed from the solution. To elute and concentrate the viral suspension, the membranes were covered with about $5 \mathrm{ml}$ of formulation buffer and incubated for $5 \mathrm{~min}$ at room temperature. Amicon filters were centrifuged at $2,600 \mathrm{~g}$ at $4{ }^{\circ} \mathrm{C}$ for approximately $1.5 \mathrm{~min}$ until around $0.5 \mathrm{ml}$ of the formulation buffer with AAV-hACE2 particles was left. The eluate was transferred into sterile 1.5 -ml tubes, quantified and stored at $4{ }^{\circ} \mathrm{C}$ for up to 2 weeks for short-term in vivo application or at $-80^{\circ} \mathrm{C}$ for long-term storage.

AAV-hACE2 titration by qPCR. The protocol for quantification and determination of the number of genome-containing particles of AAV-hACE2 was adapted from a previous publication ${ }^{39}$, using qPCR. Purified AAV-hACE2 particles were treated with DNase I (no. EN0521, Thermo Scientific) to eliminate contaminating plasmid DNA. Serial dilutions of a AAV-hACE2 viral suspension were used as template in two separate reactions, one detecting viral inverted terminal repeat (ITR) sequences (ITR_Fw: 5'-GGAACCCCTAGTGATGGAGTT-3', ITR_Rv: 5'-CGGCCTCAGTGAGCGA-3') and the second hACE2 (hACE2_Fw: 5'-CCATTGGTCTTCTGTCACCCG-3', hACE2_Rv: 5'-AGACCATCCACCTCCACTTCTC-3'). Data analysis was performed using the LightCycler 480 Software, version 1.5 . AAV concentration (the number of viral genomes in $1 \mu$ l of AAV sample) was determined by comparison to standard curves of defined concentrations of AAV-hACE2 vector. Each qPCR run was performed in triplicate; six serial dilutions of the AAV-hACE2 vector were used as positive controls and standards

\section{Mouse experiments}

This study was carried out in strict accordance with the Czech national laws and guidelines on the use of experimental animals and protection of animals against cruelty (Animal Welfare Act No. 246/1992 Coll.). The protocol was approved by the Committee on the Ethics of Animal Experiments of the Institute of Parasitology, Institute of Molecular Genetics of the Czech Academy of Sciences, and of the Departmental Expert Committee for the Approval of Projects of Experiments on Animals of the Academy of Sciences of the Czech Republic (permits $82 / 2020$ and $101 / 2020$ ).

Application of AAV-hACE2 viral particles to mice. Thirteen-tofifteen-week-old C57BL/6NCrl female mice were anaesthetized by 
intraperitoneal injection of ketamine and xylazine $(0.1 \mathrm{mg}$ per g body weight) (Biopharm) and $0.01 \mathrm{mg}$ per g (Bioveta), respectively). Viral particles containing AAV-hACE2 were diluted to a final concentration of $4 \times 10^{9}$ genome copies in $40 \mu \mathrm{l}$ of PBS. This volume was applied to mice by forced inhalation. The tip of the nose was gently clipped with tweezers and the tongue gently pulled out. After the mouse started breathing through the oral cavity, $40 \mu \mathrm{l}$ of viral suspension were applied by a $200-\mu$ l pipette tip into the oral cavity and inhaled by the mouse through the trachea into the lungs.

To access whether hACE2 was expressed in lungs, lung tissue was collected and analysed by western blot in RIPA buffer, supplemented with AEBSF protease inhibitor (AppliChem, no. A1421) and cOmplete Mini Protease Inhibitor Cocktail (Sigma Aldrich, no. 04693124001) 1, 2 and 4 weeks after application. Expression of hACE2 in AAV-hACE2 transduced mice was compared to nontreated C57BL/6NCrl mice. Histone $\mathrm{H} 3$ antibody (cat. no.: ab1791, Abcam, lot: GR3237685-2; 1:1,000 dilution) was used as a loading control.

Mouse infection. SARS-CoV-2 (strain SARS-CoV-2/human/Czech Republic/951/2020, isolated from a clinical sample at the National Institute of Public Health, Prague), provided by J. Weber, was used for mouse infection. The virus was passaged in Vero E6 cells five times before its use in this study.

At least 7 days after application of the AAV-hACE2 virus particles, mice were infected intranasally with SARS-CoV- $2\left(1 \times 10^{4}\right.$ plaque-forming units) in a total volume of $50 \mu \mathrm{l}$ DMEM. Mice were monitored and weighted daily over an eight-day period. Treated mice were injected intraperitoneally with either $150 \mu \mathrm{g}$ of antibodies $24 \mathrm{~h}$ before the infection or $250 \mu \mathrm{g} 12 \mathrm{~h}$ after infection. Mice were killed at the indicated times after infection and their tissues collected for analysis.

Measurement of the viral burden. Tissues were weighed and homogenized using Precellys 24 (Bertin Technologies) and prepared as 20\% $(\mathrm{w} / \mathrm{v})$ suspension in DMEM containing $10 \%$ newborn calf serum. The homogenates were clarified by centrifugation at 5,000 $\mathrm{g}$ and the supernatant medium was used for plaque assay and viral RNA isolation.

Plaque assays were performed in Vero E6 cells (ATCC CRL-1586; mycoplasma-free) grown at $37^{\circ} \mathrm{C}$ and $5 \% \mathrm{CO}_{2}$ in DMEM (no. LM-D1112/500, Biosera) supplemented with $10 \%$ FBS (no. FB-1001G/500; Biosera), and $100 \mathrm{U} \mathrm{ml}^{-1}$ penicillin, $100 \mathrm{\mu g} \mathrm{ml}^{-1}$ streptomycin (Antibiotic Antimycotic Solution; no. A5955; Sigma), and 1\% L-glutamine (no. XC-T1755/100; Biosera) using a modified version of a previously published protocol ${ }^{40}$. In brief, serial dilutions of virus were prepared in 24-well tissue culture plates and cells were added to each well $\left(0.6 \times 10^{5}-\right.$ $1.5 \times 10^{5}$ cells per well). After $4 \mathrm{~h}$, the suspension was overlaid with $1.5 \%$ (w/v) carboxymethylcellulose (no. C4888; Sigma) in DMEM. Following a 5-day incubation at $37^{\circ} \mathrm{C}$ and $5 \% \mathrm{CO}_{2}$, plates were washed with phosphate-buffered saline and the cell monolayers were stained with naphthol blue black (no. 195243; Sigma). The virus titre was expressed as plaque-forming units per $\mathrm{ml}$.

RNA was isolated from tissue homogenates using the QIAmp Viral RNA mini kit (no.52906; Qiagen) following manufacturer's instructions.

Viral RNA was quantified using EliGene COVID19 Basic a RT (no. 90077-RT-A; Elisabeth Pharmacon) according to the manufacturer's protocol. A calibration curve was constructed from four dilutions of a sample that was quantified using Quanty COVID-19 kit (no. RT-25; Clonit), according to the recommendations from the manufacturer. All real-time PCR reactions were performed using a LightCycler 480 (Roche).

For sequencing, isolated RNA was used as a template for one-step RT-PCR (Qiagen OneStep RT-PCR Kit; no. 210212; Qiagen) with primers specific for SARS-CoV-2 RBD sequence (SARS-CoV-2_seq FW: 5'-GCACTTGACCCTCTCTCAGAAAC-3'; SARS-CoV-2_seq_RV: 5'-GACTCAGTAAGAACACCTGTGCC-3'). The reaction mixture (final volume $25 \mu \mathrm{l}$ ) contained $5 \mu \mathrm{l}$ of QIAGEN OneStep RT-PCR buffer, $1 \mu \mathrm{l}$ of
dNTP mix, $5 \mu$ l of $5 \times$ Q-solution, $1 \mu$ l of QIAGEN OneStep RT-PCR enzyme mix, $6 \mu$ l of RNase-free water, $1.5 \mu$ l of each primer (stock concentration, $0.01 \mathrm{mM}$ ) and $4 \mu \mathrm{l}$ of template RNA. The cycling conditions were as follows: reverse transcription $\left(30 \mathrm{~min}\right.$ at $50^{\circ} \mathrm{C}$ ), initial PCR activation ( 15 min at $95^{\circ} \mathrm{C}$ ), 3-step cycling: 40 cycles of $94^{\circ} \mathrm{C}$ for $30 \mathrm{~s}, 52.6^{\circ} \mathrm{C}$ for $30 \mathrm{~s}$, and $72{ }^{\circ} \mathrm{C}$ for $1 \mathrm{~min}$, followed by final extension $\left(10 \mathrm{~min}\right.$ at $\left.72{ }^{\circ} \mathrm{C}\right)$. The PCR products were visualized in a $1.7 \%$ agarose gel in Tris-acetate-EDTA buffer. The amplified DNA was purified by using Wizard SV Gel and PCR Clean-Up System (no. A9285; Promega), according to the recommendations of the manufacturer.

The purified DNA was prepared for sequencing (Sanger method) by a commercial service (Eurofins Genomics) with the following conditions (final volume $17 \mu \mathrm{l}$ ): $15 \mu \mathrm{l}$ of PCR product with a concentration of $5 \mathrm{ng} \mathrm{\mu l}^{-1}$ and $2 \mu \mathrm{l}$ of primer with a concentration of $10 \mu \mathrm{M}$. The sequencing data were analysed using BioEdit Sequence Alignment Editor, version 7.2.0.

Histology and immunohistochemistry. Lungs were fixed in 4\% PFA. Tissues were processed using a Leica ASP6025 automatic vacuum tissue processor and embedded in paraffin using a Leica EG1150 H+C embedding station. Two- $\mu \mathrm{m}$ sections were prepared using a Leica RM2255 rotary microtome and sections were stained with H\&E using Leica ST5020 + CV5030 stainer and coverslipper.

To assess the presence of macrophages, a rabbit anti-mouse F4/80 monoclonal antibody (D2S9R XPR rabbit monoclonal antibody, cat. no. 70076, Cell Signaling Technology, lot 5, RRID AB_2799771) was used at 1:800 dilution as primary antibody. The histological sections (thickness $4-5 \mu \mathrm{m}$ ) were deparaffinized in a Multistainer Leica ST5020 (Leica Biosystems). Antigens were retrieved by heating the slides in citrate buffer pH 6 (Zytomed Systems). Endogenous peroxidase was neutralized with $3 \% \mathrm{H}_{2} \mathrm{O}_{2}$. Sections were incubated for $1 \mathrm{~h}$ at room temperature with a 1:800 dilution of the primary antibody. After washing they were incubated with anti-rabbit secondary antibody conjugated with HRP (Zytomed, cat. no. ZUC 032-100, lot A0880-4; no dilution). Staining of the sections was developed with a diaminobenzidine substrate kit (DAKO, Agilent) and sections were counterstained with Harris haematoxylin (Sigma Aldrich, Merck) in a Multistainer Leica.

\section{Biosafety statement}

All work with infectious SARS-CoV-2 was performed in biosafety level 3 facilities at the Institute of Parasitology, Biology Centre of the Czech Academy of Science (Ceske Budejovice) and Veterinary Research Institute (Brno) using appropriate powered air-purifying, positive pressure respirators and protective equipment.

\section{Reporting summary}

Further information on research design is available in the Nature Research Reporting Summary linked to this paper.

\section{Data availability}

The data that support the findings of this study are available within the Article and its Supplementary Information. Any other data are available from the corresponding author upon reasonable request. Published data were taken from GenBank (https://www.ncbi.nlm.nih. gov/genbank/), UniProt (https://www.uniprot.org/), PDB (https://www. rcsb.org/) and the ViPR database (https://www.viprbrc.org/). Source data are provided with this paper.

31. Bardelli, M. et al. A bispecific immunotweezer prevents soluble PrP oligomers and abolishes prion toxicity. PLoS Pathog. 14, e1007335 (2018).

32. Fu, B. et al. ALMOST: an all atom molecular simulation toolkit for protein structure determination. J. Comput. Chem. 35, 1101-1105 (2014).

33. Yang, J. et al. The I-TASSER suite: protein structure and function prediction. Nat. Methods 12, 7-8 (2015)

34. Schrodinger. The PyMOL Molecular Graphics System, Version 1.8 (Schrodinger 2015).

35. Van Der Spoel, D. et al. GROMACS: fast, flexible, and free. J. Comput. Chem. 26, 1701-1718 (2005). 
36. Wang, Z. et al. mRNA vaccine-elicited antibodies to SARS-CoV-2 and circulating variants. Nature (2021).

37. Percivalle, E. et al. West Nile or Usutu virus? A three-year follow-up of humoral and cellular response in a group of asymptomatic blood donors. Viruses 12, 157 (2020).

38. Zolotukhin, S. et al. Recombinant adeno-associated virus purification using novel methods improves infectious titer and yield. Gene Ther. 6, 973-985 (1999).

39. Aurnhammer, C. et al. Universal real-time PCR for the detection and quantification of adeno-associated virus serotype 2-derived inverted terminal repeat sequences. Hum. Gene Ther. Methods 23, 18-28 (2012).

40. De Madrid, A. T. \& Porterfield, J. S. A simple micro-culture method for the study of group B arboviruses. Bull. World Health Organ. 40, 113-121 (1969).

Acknowledgements D.F.R., L.V., Q.P.-H., F.Baldanti and L.C. have received funding from the European Union's Horizon 2020 research and innovation programme under grant agreement no. 101003650 . This work was also supported by SNF grant 31003A_182270 (L.V.); Lions Club Monteceneri (L.V.); George Mason University Fast Grant and IRB start-up funds (D.F.R.); NIH U01 Al151698 for the United World Antiviral Research Network (UWARN) (D.F.R. and M.C.N.); NIH grant P01-Al138398-S1 (M.C.N. and P.J.B.); 2U19A1111825 (M.C.N. and D.F.R.); the Caltech Merkin Institute for Translational Research and P50 Al150464 (P.J.B.); R37-Al64003 (P.D.B.); and R01AI78788 (T.H.); P.D.B. and M.C.N. are Howard Hughes Medical Institute Investigators. The study was also supported by the Czech Academy of Sciences and Czech Ministry of Agriculture (RVO 68378050 (R.S.) and RVO0518 (D.R.)); Czech Ministry of Education, Youth and Sports and the European Regional Development Fund (LM2018126; CZ.1.05/2.1.00/19.0395 and CZ.1.05/1.1.00/02.0109 (R.S.) and CZ.02.1.01/0.0/0.0/15_003/0000495 (D.R.)); Czech Science Foundation (2O-14325S (D.R.)); the Bulgari Women \& Science Fellowship in COVID-19 Research (F. Muecksch); the EU Joint Research Centre Exploratory Research program ('NanoMicrobials';
D. Magri); and by Ricerca Finalizzata from Ministry of Health, Italy (grant no. GR-201302358399 (A.P.)). We are grateful for the high-performance computing resources that were provided by S. Bassini of CINECA to M. Hust, F. Bertoglio, F. Bognuda and E. Restivo. We thank V. Zatecka, V. Martinkova and L. Kutlikova for technical assistance; and V. Babak for help with statistical analyses. We are grateful to the late F. Diederich for their mentorship.

Author contributions R.D.G, M. Pedotti, L.S., F. Muecksch, J.C.C.L., F. Mazzola, D. Magrì, I.C., E.P., S.D.G., M. Palus, D. Mehn, S. Gioria, C.O.B., F. Bianchini, J.C.S., F.G. and S. Gaiarsa designed and carried out experiments and analysed results, and produced plasmids, antibodies and viral proteins. P.N., T.M., J.H., V.H, B.M., N.P., A.F., J.T., V.I., M. Palus, D.Z., P.B., I.B., P.S. and D.R., performed mouse experiments and analysed the results. L.V., D.F.R., D.R., Q.P.-H., F. Baldanti, A.P., L.C., P.J.B., M.C.N., P.D.B. and T.H. conceived and designed study and experiments, and analysed the results. P.N., T.M., R.N., O.P., J.P., J.R. and R.S. conceived and designed the mouse model. L.V., D.F.R., D.R. and R.D.G. wrote the manuscript, with input from all co-authors.

Competing interests The Institute for Research in Biomedicine has filed a provisional European patent application in connection with this work, on which L.V. is inventor (PCT/EP2020/085342). The Rockefeller University has filed a provisional US patent application (US 63/021,387) on coronavirus antibodies, on which D.F.R. and M.C.N. are inventors.

Additional information

Supplementary information The online version contains supplementary material available at https://doi.org/10.1038/s41586-021-03461-y.

Correspondence and requests for materials should be addressed to D.F.R., D.R. or L.V.

Peer review information Nature thanks Stanley Perlman and the other, anonymous, reviewer(s) for their contribution to the peer review of this work.

Reprints and permissions information is available at http://www.nature.com/reprints. 


\section{Article}

a

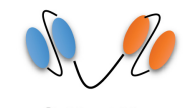

CoV-scB1

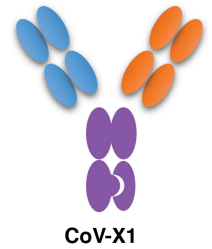

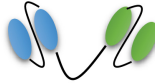

CoV-scB2

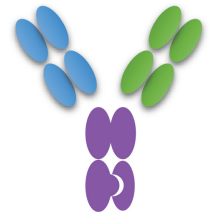

CoV-X2

Extended Data Fig. 1 | Neutralization of SARS-CoV-2 pseudovirus by bispecific antibodies. a, Schematic of the four bispecific antibodies; two in an scFv format and two in a IgG-like CrossMAb format with knob-in-hole. The parental monoclonal antibodies that form the bispecific antibodies are colour-coded: blue, C135; orange, C144; green, C121; and purple, Fc region.

b, All four constructs neutralize SARS-CoV-2 pseudovirus in vitro at

b

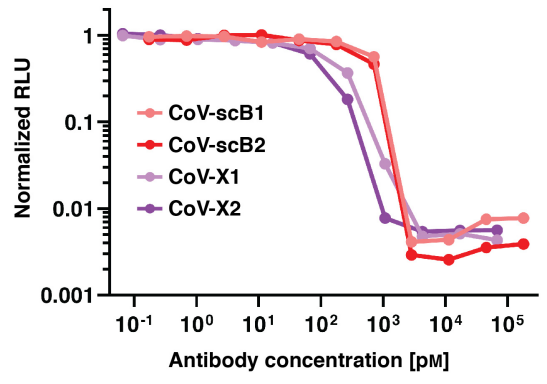

sub-nanomolar concentrations: $\mathrm{IC}_{50}$ of $0.13,0.04,0.74$ and $0.53 \mathrm{nM}$ for CoV-X1, CoV-X2, CoV-scB1 and CoV-scB2, respectively. Normalized relative luminescence values (which correlate to infection) are reported versus antibody concentration, as previously detailed ${ }^{15}$. Mean of two replicates from one representative experiment is shown. 
a
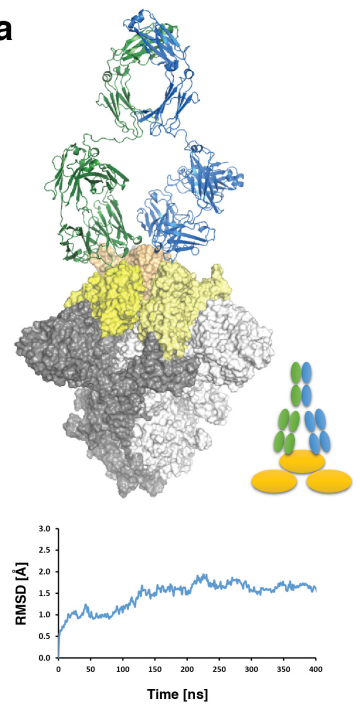

e

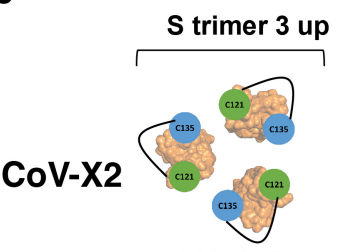

3 bivalent b
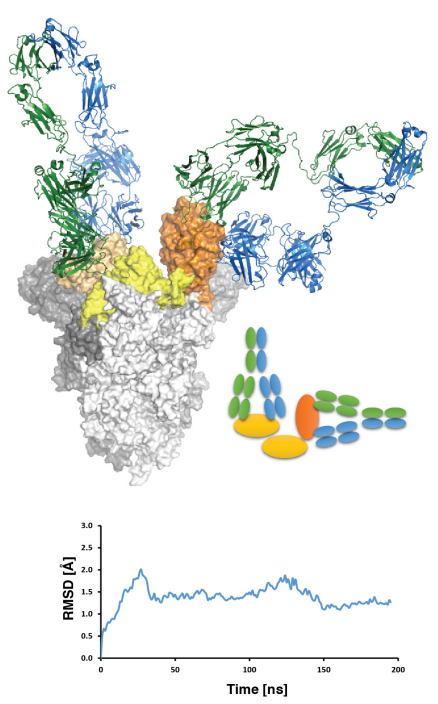

S trimer 2 up

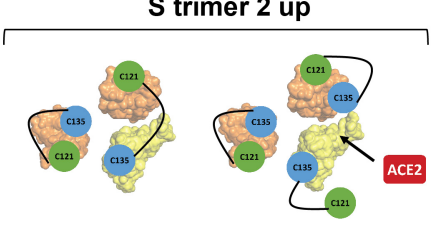

$\underline{2 \text { bivalent }}$ c
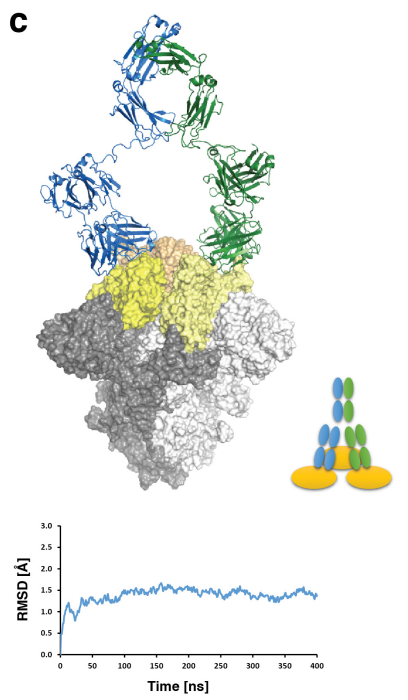

S trimer 1 up

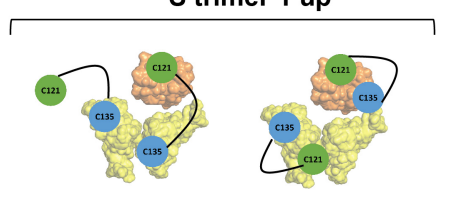

1 biv / 1 mono d
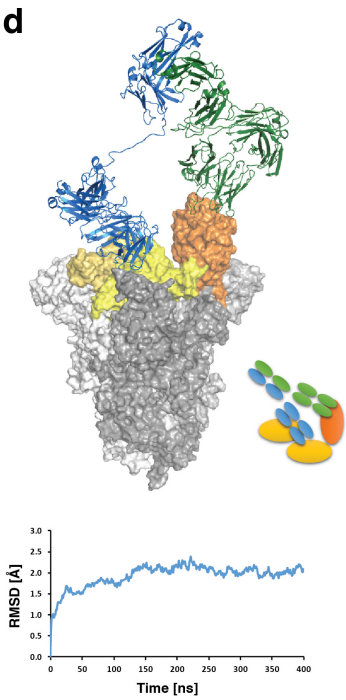

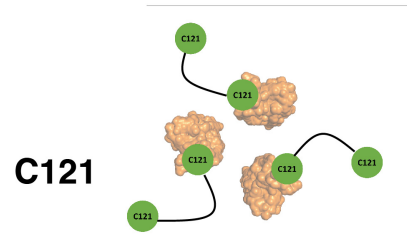

3 monovalent

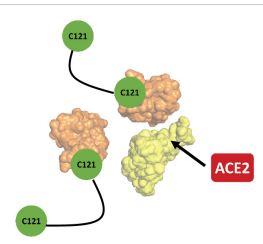

2 monovalent

2 monovalent

1 biv / 1 mono

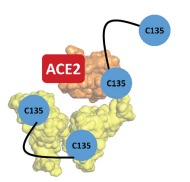

1 biv / 1 mono
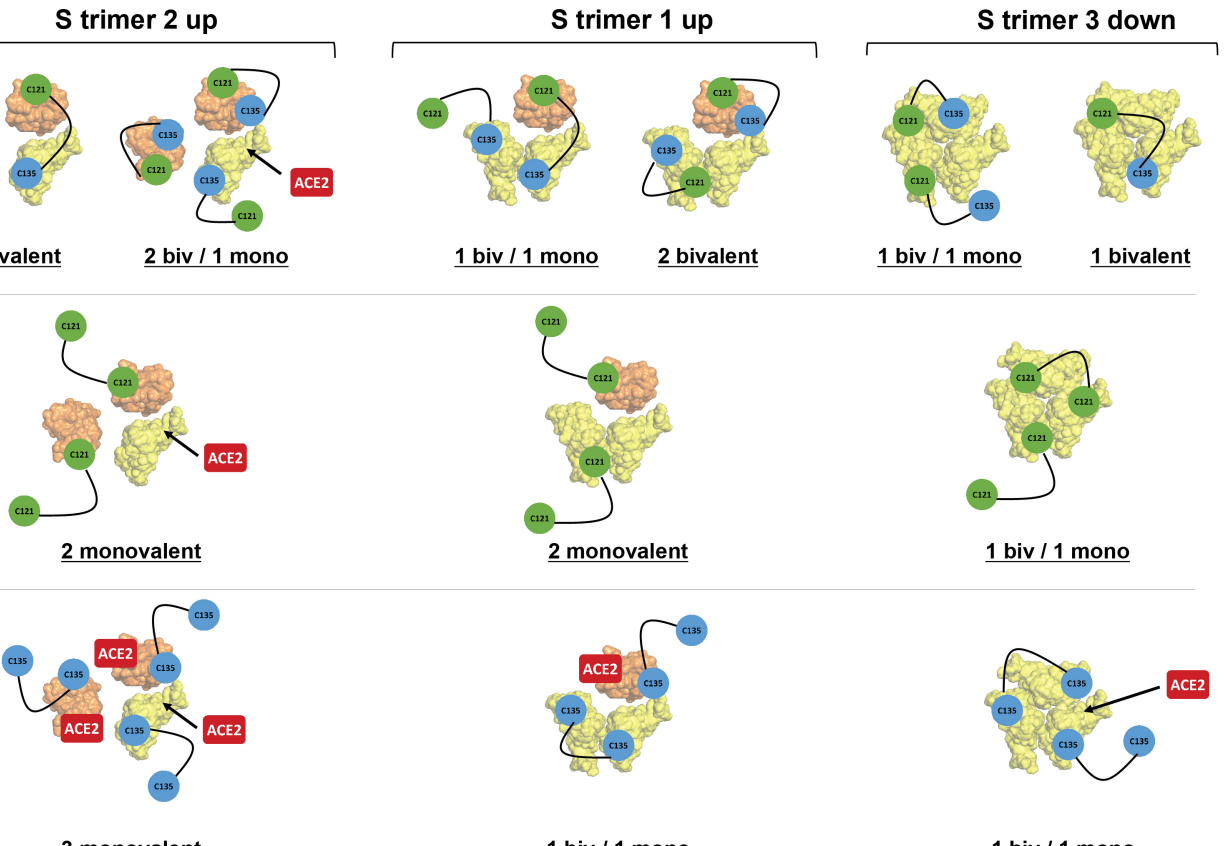

Extended Data Fig. 2 | CoV-X2 engages its epitopes on all RBD

conformations on the $S$ trimer. a-d, Molecular dynamics simulations of the complex between the CoV-X2 bispecific antibody and S trimers with RBD in either all down, all up or mixed up/down conformations show that $\mathrm{CoV}-\mathrm{X} 2 \mathrm{can}$ engage a single RBD with both arms $(\mathbf{a}, \mathbf{b})$, two adjacent RBDs in the down conformation (c) and two RBDs in the up and down conformation (b, d). The complexes were subjected to up to 400 ns of fully atomistic molecular dynamics simulations to assess feasibility and stability of the bound conformations. Root-mean-squared deviations (r.m.s.d.) values are shown to indicate structural stability. Strimer is in shades of grey, RBDs in yellow (down conformation) and orange (up), the $\mathrm{C} 121$ and $\mathrm{C} 135$ moieties of $\mathrm{CoV}-\mathrm{X} 2$ are in

green and blue, respectively.e, Schematic of the computationally predicted binding modes of $\mathrm{CoV}-\mathrm{X} 2, \mathrm{C} 121 \mathrm{IgG}$ and $\mathrm{C} 135 \mathrm{IgG}$ on the S trimer, coloured as in a-d. Antibodies are represented by connected circles; ACE2 is in red on the RBD if it can bind directly to a given conformation; and an arrow points to the RBD if ACE2 binding is achieved after an allowed switch to the up conformation. For example, in the three-up conformation (left), $\mathrm{CoV}-\mathrm{X} 2$ can engage all the RBDs with bivalent binding, whereas $\mathrm{C} 121$ and $\mathrm{C} 135$ can achieve only monovalent binding. C135 binding does not prevent interaction with ACE2. The situation is similar in the other $\mathrm{S}$ conformations (two-up and one-down, two-down and one-up, and three-down); only the bispecific antibody achieves bivalent interaction and prevents ACE2 access in all conformations. 


\section{Article}

\section{a}
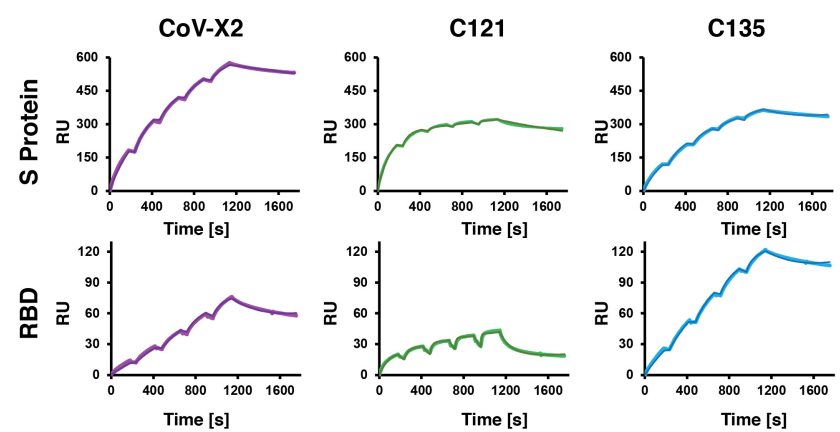

\begin{tabular}{|c|c|c|c|c|}
\hline \multicolumn{2}{|c|}{ o } & CoV-X2 & C121 & C135 \\
\hline \multirow{3}{*}{ 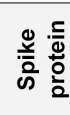 } & $k_{\mathrm{a}}\left[\cdot 10^{6} \mathrm{M}^{-1} \mathrm{~s}^{-1}\right]$ & 0.69 & 1.33 & 0.62 \\
\hline & $k_{\mathrm{d}}\left[\cdot 10^{-3} \mathrm{~s}^{-1}\right]$ & 0.12 & 0.17 & 0.13 \\
\hline & $K_{\mathrm{D}}[\mathrm{nM}]$ & 0.18 & 0.13 & 0.21 \\
\hline \multirow{3}{*}{$\begin{array}{l}\stackrel{0}{\alpha} \\
\underline{x}\end{array}$} & $k_{\mathrm{a}}\left[-10^{6} \mathrm{M}^{-1} \mathrm{~s}^{-1}\right]$ & 0.58 & 1.30 & 0.20 \\
\hline & $k_{\mathrm{d}}\left[\cdot 10^{-3} \mathrm{~s}^{-1}\right]$ & 1.35 & 3.15 & 0.12 \\
\hline & $K_{\mathrm{D}}[\mathrm{nM}]$ & 2.35 & 2.40 & 0.59 \\
\hline
\end{tabular}

Extended Data Fig. 3 | CoV-X2 and its parental monoclonal antibodies bind recombinant, isolated RBD and $S$ trimer with low nanomolar affinity.

a, Representative SPR traces from which the data in $\mathbf{b}$ were derived. b, Kinetic parameters for the binding of C121 IgG, C135 IgG and CoV-X2 to the S trimer and RBD. 
a

\begin{tabular}{|l|c|c|c|}
\hline & $\begin{array}{c}\text { CoV-X2 } \\
\boldsymbol{K}_{\mathrm{D}}[\mathrm{nM}]\end{array}$ & $\begin{array}{c}\text { C121 } \\
\boldsymbol{K}_{\mathrm{D}}[\mathrm{nM}]\end{array}$ & $\begin{array}{c}\text { C135 } \\
\boldsymbol{K}_{\mathrm{D}}[\mathrm{nM}]\end{array}$ \\
\hline Wild-type & 2.3 & 2.4 & 0.6 \\
\hline N331A & 2.1 & 0.3 & 0.4 \\
\hline E340K & 1.4 & 1.9 & $<0.1$ \\
\hline T345A & 1.2 & 1.4 & 0.2 \\
\hline V367F & 2.4 & 2.1 & 0.5 \\
\hline R408I & 1.9 & 2.1 & 0.7 \\
\hline K417A & 1.3 & 0.7 & 0.3 \\
\hline W436R & 2.7 & 3.3 & 2.7 \\
\hline K444E & 1.1 & 2.8 & No binding \\
\hline E484A/F486A & 7.8 & No binding & 1.7 \\
\hline F490A & 2.9 & 35 & 1.5 \\
\hline T500A & 1.5 & 1.5 & 1.0 \\
\hline Y505A & 1.4 & 1.6 & 0.6 \\
\hline $\begin{array}{l}\text { R346T/A372T/1402V/ } \\
\text { K417R/Q498H/H519K } \\
\text { (Pangolin GD) }\end{array}$ & 2.5 & 0.5 & No binding \\
\hline D614G & 3.2 & 0.6 & 1.6 \\
\hline
\end{tabular}

Extended Data Fig. 4 | CoV-X2 binds with low-nanomolar affinity to $S$ mutants, including some mutants that are not recognized by the parental monoclonal antibodies C121 and C135. a, SPR-derived binding affinities of

b

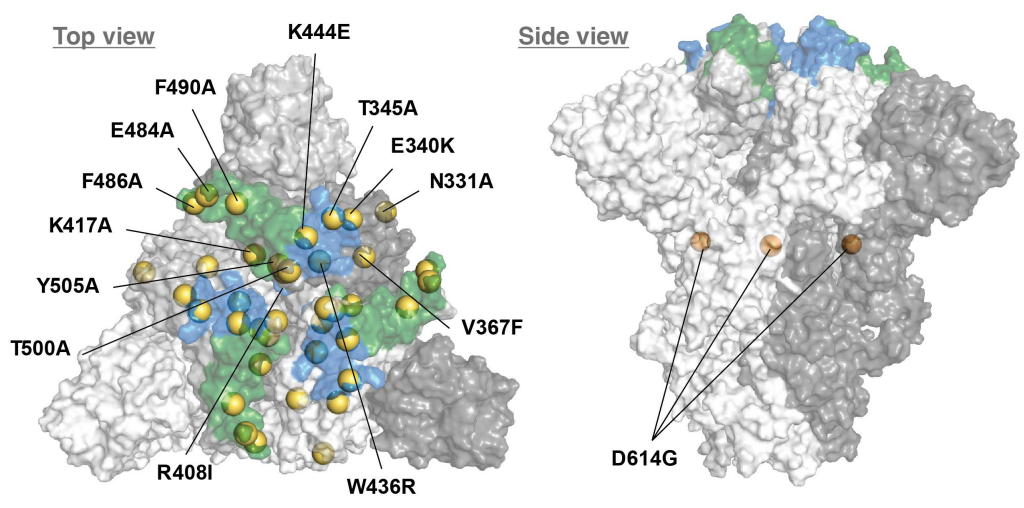

CoV-X2, C121 IgG and C135 IgG to several S trimer mutants. b, Mutations tested in a are indicated by yellow spheres on the surface representation of the S trimer. The epitopes of C121 (green) and C135 (blue) are shown. 


\section{Article}

a

Wild-type

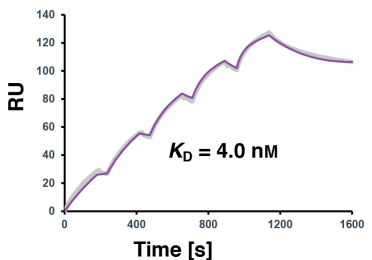

b

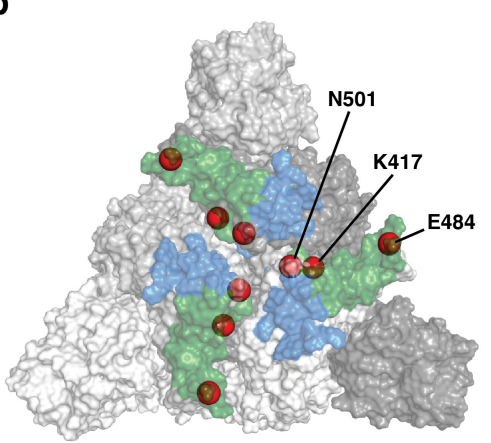

Extended Data Fig. 5 | Efficacy of CoV-X2 against the B.1.1.7 and B.1.351

variants. a, SPR traces showing binding of $\mathrm{CoV}-\mathrm{X} 2$ to the $\mathrm{RBD}$ corresponding to wild-type SARS-CoV-2 and the B.1.1.7 and B.1.351 variants. b, Residues mutated in the variants are shown as red spheres on the surface representation of the S trimer. The epitopes of C121 (green) and C135 (blue) are shown.

C
B.1.1.7
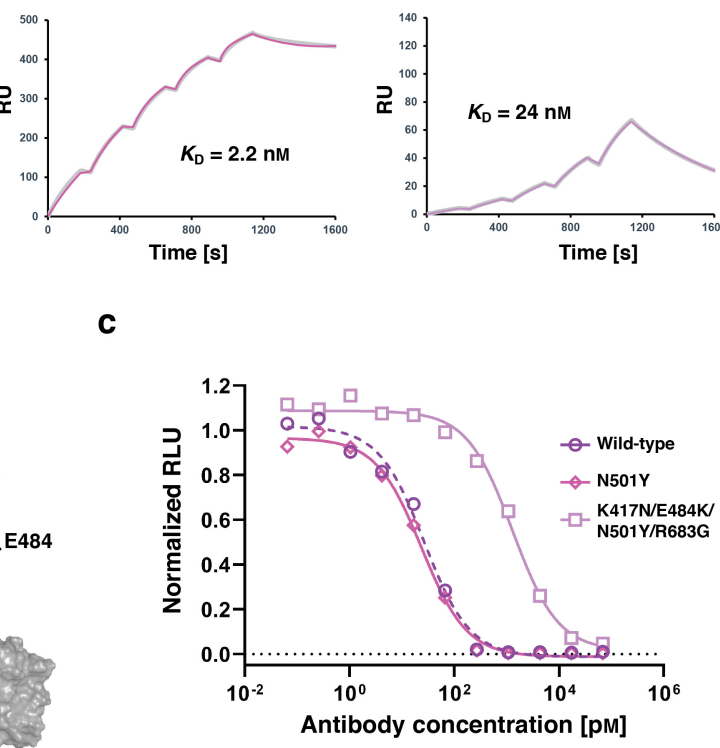

c, Neutralization of SARS-CoV-2 pseudoviruses expressing wild-type $S$, S(N501Y) and S(K417N/E484K/N501Y/R683G) (corresponding to substitutions in the RBD in the B.1.351 variant (Fig. 1h)) by CoV-X2. Mean of two experiments is shown.

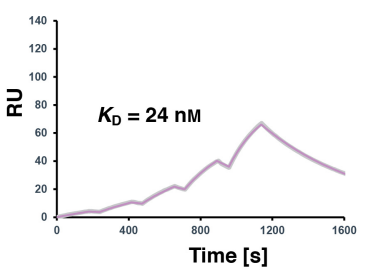




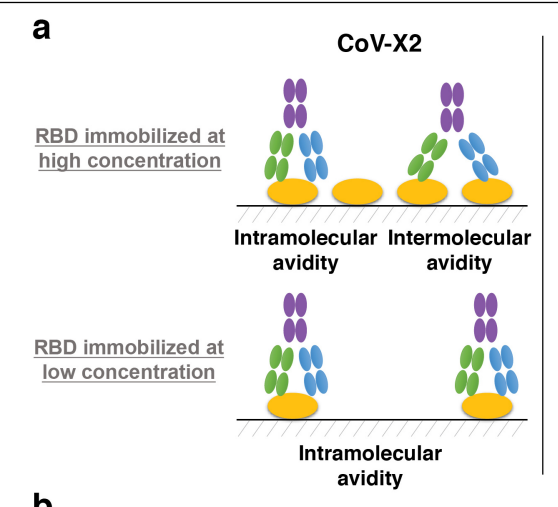

b
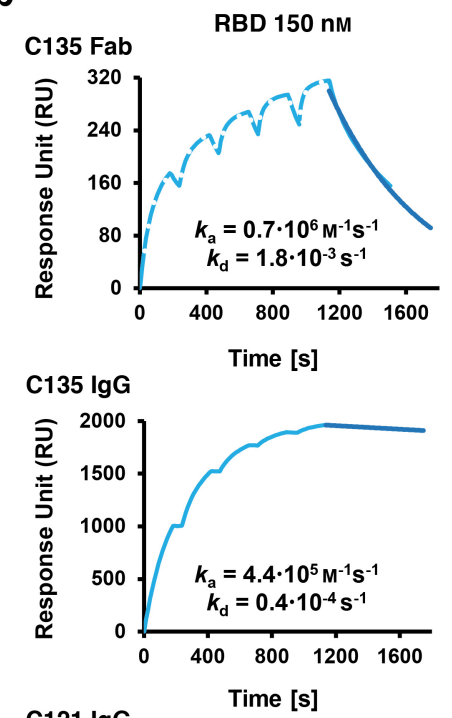

C121 IgG
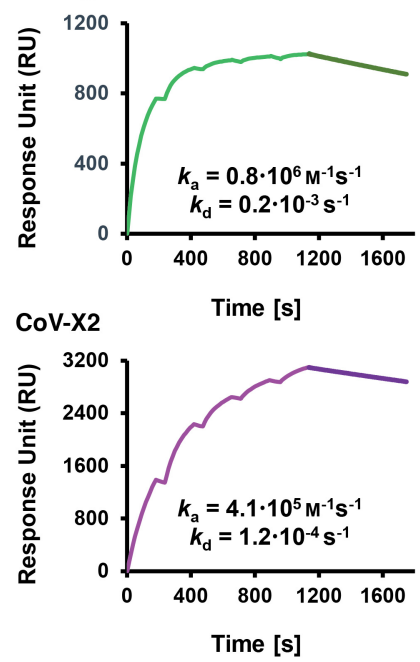

IgGs

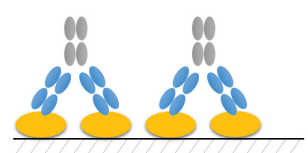

Intermolecular avidity

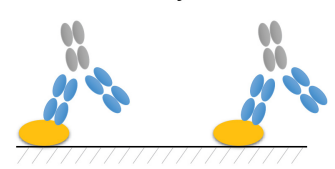

No avidity

\begin{tabular}{|c|c|c|c|c|c|}
\hline & \multicolumn{4}{|c|}{ Immobilized on the chip } & \\
\hline & RBD $150 \mathrm{nM}$ & RBD $75 \mathrm{nM}$ & RBD $15 \mathrm{nM}$ & RBD $5 \mathrm{nM}$ & \\
\hline C135 Fab & 1.0 & 1.2 & 1.7 & 1.7 & \multirow{4}{*}{$\begin{array}{c}\boldsymbol{k}_{\mathbf{a}} \\
\text { Normalized } \\
k_{\mathrm{a}}\left(\mathrm{X}_{\mathrm{i}} \mathrm{nM}\right) / k_{\mathrm{a}}(150 \mathrm{nM})\end{array}$} \\
\hline C135 IgG & 1.0 & 1.1 & 1.0 & 0.9 & \\
\hline C121 IgG & 1.0 & 1.1 & 3.1 & 2.6 & \\
\hline CoV-X2 & 1.0 & 1.4 & 1.8 & 2.0 & \\
\hline C135 Fab & 1.0 & 1.1 & 1.0 & 0.9 & \multirow{4}{*}{$\begin{array}{c}\boldsymbol{k}_{\mathbf{d}} \\
\text { Normalized } \\
k_{\mathrm{d}}\left(\mathrm{X}_{\mathrm{i}} \mathrm{nM}\right) / k_{\mathrm{d}}(150 \mathrm{nM})\end{array}$} \\
\hline C135 IgG & 1.0 & 2.3 & 9.1 & 18.8 & \\
\hline C121 IgG & 1.0 & 3.2 & 9.0 & 18.5 & \\
\hline CoV-X2 & 1.0 & 1.3 & 3.2 & 5.7 & \\
\hline
\end{tabular}

RBD 75 nM

RBD $15 \mathrm{nM}$

RBD 5 nM
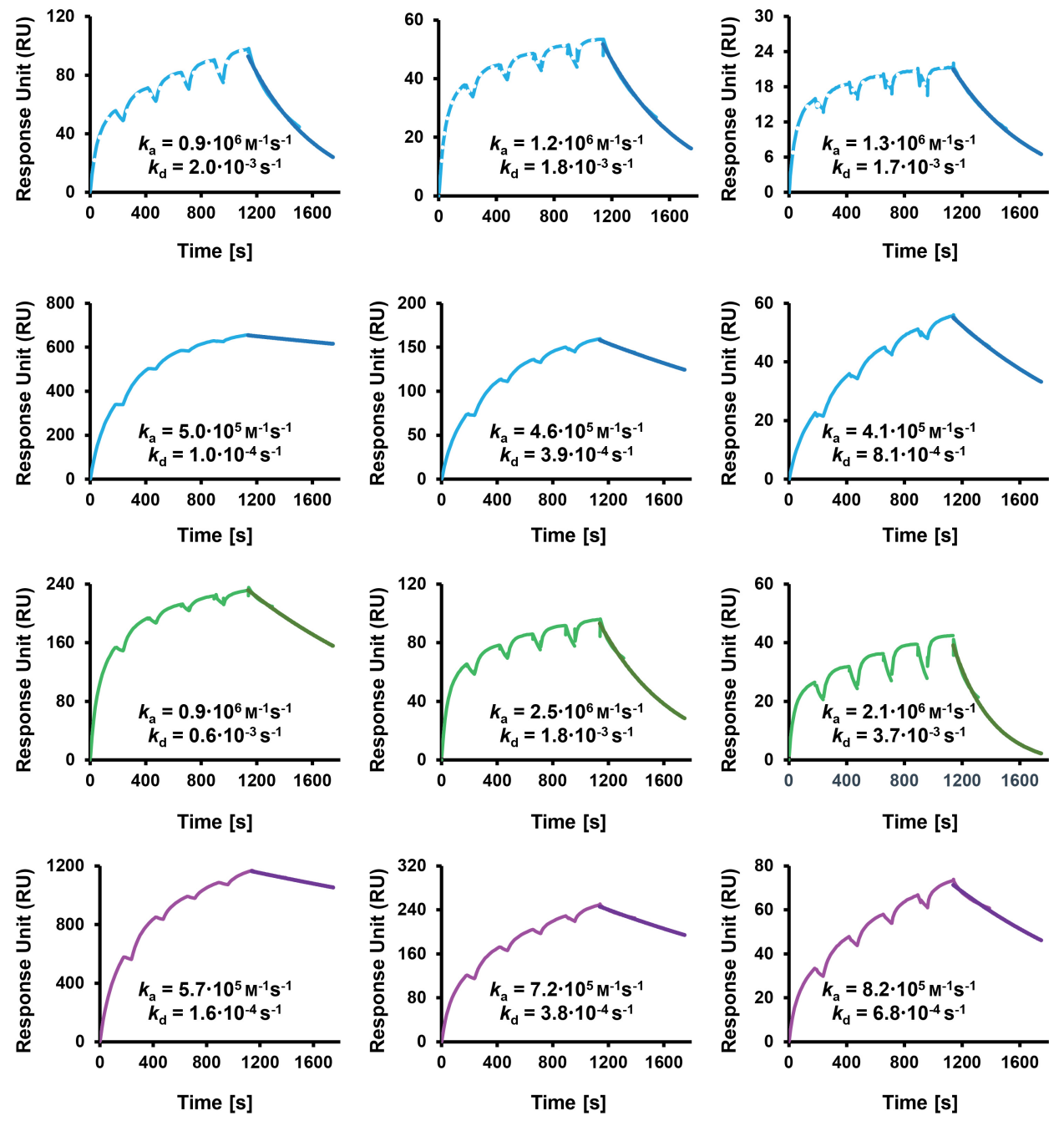

affected by avidity. $\mathbf{b}$, Experimental confirmation that $\mathrm{CoV}-\mathrm{X} 2$ engages bivalently on a single RBD. SPR traces used to determine $k_{\mathrm{a}}$ and $k_{\mathrm{d}}$ of monoclonal antibodies, Fab and the bispecific antibody at different concentrations of immobilized RBD (Fig. 1d) are shown.c, Table summarizing the SPR results plotted in Fig. 1d. $k_{\mathrm{a}}$ and $k_{\mathrm{d}}$ were normalized against the values at the highest RBD concentration. $k_{\mathrm{a}}$ and Fab $k_{\mathrm{d}}$ were unaffected by the RBD concentration, as expected. $k_{\mathrm{d}}$ became faster for the monoclonal antibodies (loss of avidity) but less so for the bispecific antibody (avidity maintained owing to simultaneous binding to two sites on a single RBD). 


\section{Article}

a
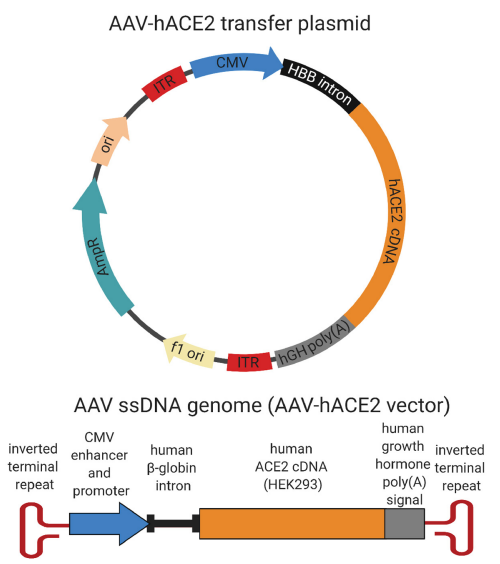

b
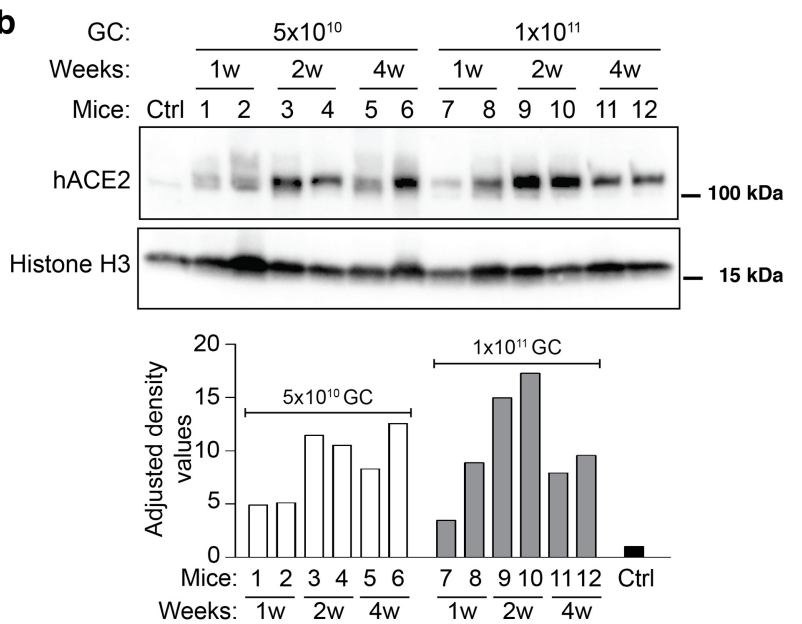

c

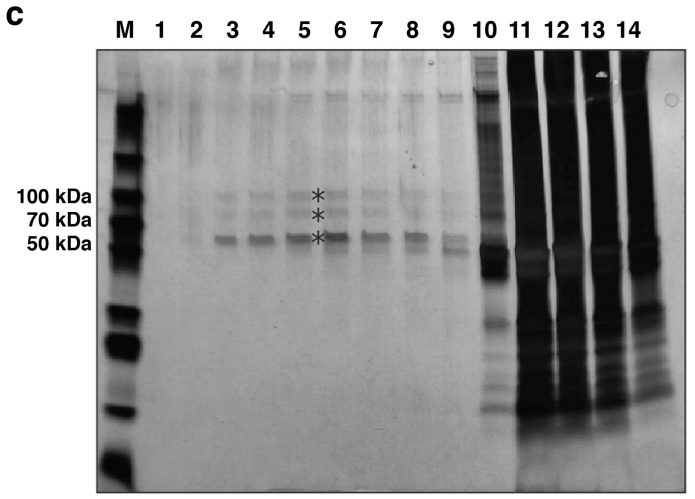

d

\begin{tabular}{|c|c|c|c|c|c|c|c|}
\hline $\begin{array}{l}\text { AAV-hACE2 } \\
\text { dilutions }\end{array}$ & Ct1 & $\mathrm{Ct} 2$ & Ct3 & Average $\mathrm{Ct}$ & $\begin{array}{c}\text { No. of GC } \\
(\log )\end{array}$ & No. of GC & $\begin{array}{c}\begin{array}{c}\text { No. of GC } \\
\text { counted from } \\
\text { dilution }\end{array} \\
\end{array}$ \\
\hline $3125000 x$ & 25.63 & 25.68 & 25.70 & 25.67 & 3.526 & $3.36 \mathrm{E}+03$ & $1.05 \mathrm{E}+10$ \\
\hline $625000 x$ & 24.70 & 24.60 & 24.70 & 24.67 & 3.874 & $7.48 \mathrm{E}+03$ & $4.67 \mathrm{E}+09$ \\
\hline $125000 x$ & 22.84 & 22.69 & 22.83 & 22.79 & 4.526 & $3.36 \mathrm{E}+04$ & $4.20 \mathrm{E}+09$ \\
\hline $25000 x$ & 20.65 & 20.61 & 20.86 & 20.71 & 5.248 & $1.77 \mathrm{E}+05$ & $4.43 \mathrm{E}+09$ \\
\hline $5000 x$ & 18.33 & 18.43 & 18.25 & 18.34 & 6.070 & $1.18 \mathrm{E}+06$ & $5.88 \mathrm{E}+09$ \\
\hline $1000 x$ & 16.15 & 16.00 & 16.05 & 16.07 & 6.858 & $7.21 \mathrm{E}+06$ & $7.21 \mathrm{E}+09$ \\
\hline & \multicolumn{2}{|c|}{$\begin{array}{c}\text { Average No. of } \\
\text { GC/reaction }\end{array}$} & & $G C / 4$ & \multicolumn{2}{|c|}{$4 \times 10^{9} \mathrm{GC}(\mu \mathrm{l})$} & (to $40 \mu$ l) \\
\hline AAV-hACE2 & \multicolumn{2}{|c|}{$6.15 \mathrm{E}+09$} & \multicolumn{2}{|r|}{$2.46 \mathrm{E}+09$} & \multicolumn{2}{|l|}{1.63} & 38.37 \\
\hline
\end{tabular}

e

SARS-CoV-2 infection

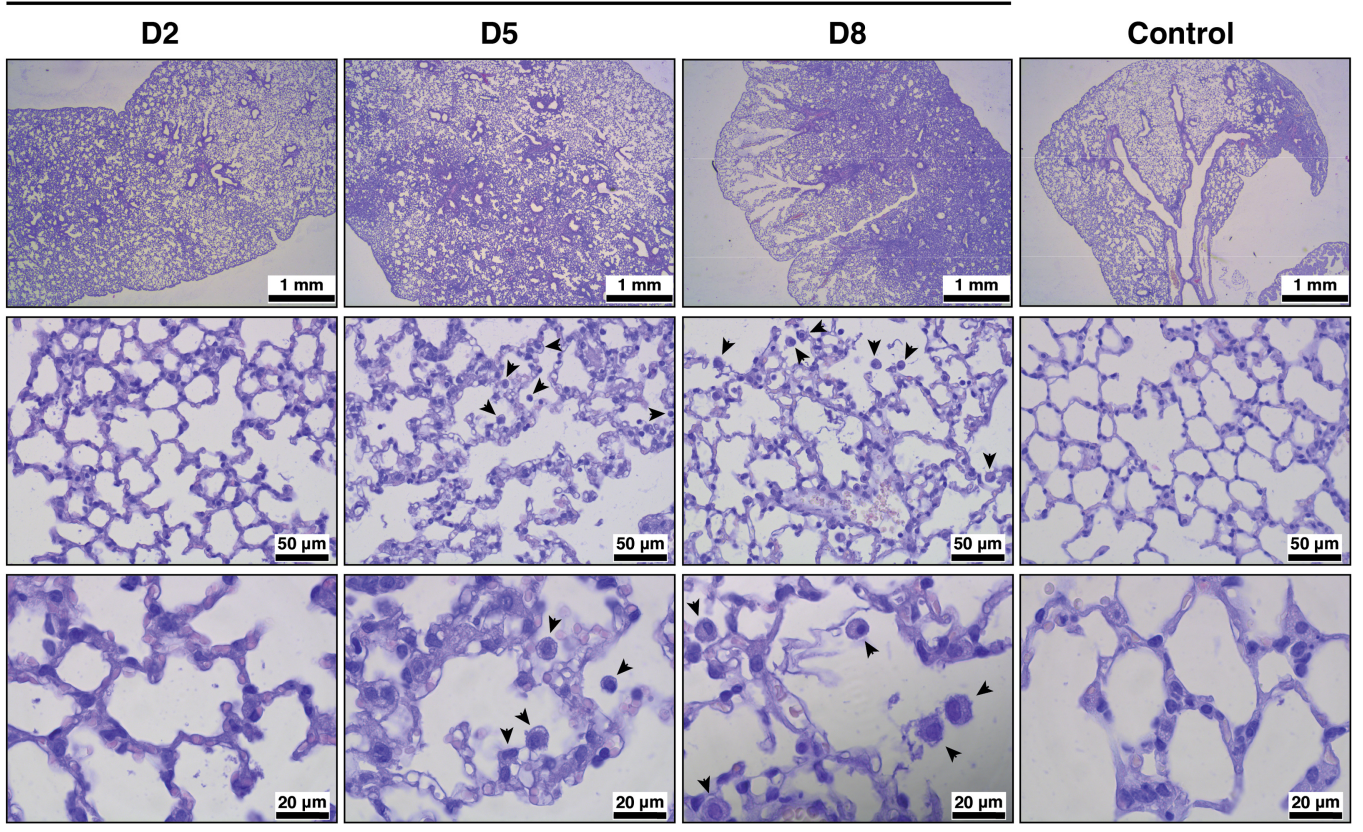

Extended Data Fig. 7 |See next page for caption. 
Extended Data Fig. 7 |Generation of the AAV-hACE2-transduced mouse model of COVID-19.a, Diagram of the AAV-hACE2 plasmid and corresponding AAV vector. b, Western blot analysis detecting hACE2 expression in the lungs of one non-transduced control mouse (ctrl) and 12 mice transduced with two doses of AAV-hACE2 viral particles $\left(5 \times 10^{10}\right.$ or $1 \times 10^{11}$ genome copies (GC)). Lung tissue was collected 1, 2 or 4 weeks (w) after transduction. Histone $\mathrm{H} 3$ was used as control for quantification (bottom). Quantitative analysis represents normalized data from membrane images (top), and was performed using ImageJ. Representative data from two independent experiments are shown. c, Preparation of concentrated AAV-hACE2. AAV-hACE2 plasmid was cotransfected with pHelper and AAV Rep/Cap 2/9n vectors into 293AAV cells (Methods). To increase viral titres, viral particles from both cell lysate and PEG-precipitated growth medium were ultracentrifuged in a discontinuous iodixanol gradient. The silver-stained SDS-PAGE gel shows 14 consecutive fractions: fractions $1-9$ represent enriched AAV fractions used for experiments, and fractions 10-14 are contaminated with proteinaceous cell debris. Iodixanol was chosen as a density gradient medium owing to its low toxicity in vivo and its easy removal by ultrafiltration. M, protein marker; *AAV capsid proteins VP1, VP2, and VP3. Representative data from two independent experiments are shown.d, The amount of AAV particles was estimated by RT-qPCR. The number of genome copies expressed as log was calculated from a standard curve. From one $15-\mathrm{cm}^{2}$ dish, $75 \mu$ l with $2.0 \times 10^{12}$ genome copies per $\mathrm{ml}$ were prepared, which is sufficient for hACE2 humanization of 37 mice. e, Kinetic of lung histopathology in SARS-CoV-2-infected ACE2-humanized mice. H\&E-stained sections showed inflammatory infiltrates composed of lymphocytes, macrophages, neutrophils and fibroblasts replacing the alveoli. The size of the affected areas increased over time (area of diffuse alveolar damage: control $<5-10 \%, 2 \mathrm{dpi}<10-30 \%, 5 \mathrm{dpi} 20-80 \%$ and $8 \mathrm{dpi} 50-90 \%$ ). Alveolar septa were thickened in areas that were close to infiltrates. In samples collected at 5 and $8 \mathrm{dpi}$, an increased number of activated macrophages with foamy cytoplasm (black arrowheads) was seen. AAV-hACE2-transduced, SARS-CoV-2-uninfected mice were used as control and showed no noticeable pathology. Each image is representative of two separate experiments ( $n=3$ to 5 mice per group). 


\section{Article}

a

\begin{tabular}{|c|c|c|c|c|c|}
\hline wt & mutant & Strain Name & wt & mutant & Strain Name \\
\hline Arg 403 & Lys & $\begin{array}{l}\text { /USA/VA-DCLS-0630/2020 } \\
\text { /USA/VA-DCLS-0439/2020 } \\
\text { /AUS/VIC1787/2020 } \\
\end{array}$ & \multirow{4}{*}{ Gly485 } & \multirow{4}{*}{ Arg } & $\begin{array}{l}\text { /AUS/VIC1829/2020 } \\
\text { /AUS/NIC1960/2020 } \\
\text { /AUS/VIC1693/2020 }\end{array}$ \\
\hline Lys444 & Asn & /AUS/VIC4515/2020 & & & $\begin{array}{l}\text { /AUS/NIC1660/2020 } \\
\text { /AUS/NIC1683/2020 }\end{array}$ \\
\hline & Asp & /USA/FL-BPHL-2211/2020 & & & /AUS/NIC1588/2020 \\
\hline Gly446 & Val & $\begin{array}{l}\text { /USA/MN-MDH-1430/2020 } \\
\text { /AUS/VIC913/2020 } \\
\text { /AUS/VIC6087/2020 } \\
\text { /AUS/VIC9542/2020 }\end{array}$ & & & $\begin{array}{l}\text { /AUS/VIC1565/2020 } \\
\text { /AUS/NIC1611/2020 } \\
\text { /AUS/NIC1812/2020 } \\
\text { /AUS/NIC2023/2020 } \\
\end{array}$ \\
\hline \multirow{4}{*}{ Leu452 } & Gln & /USA/VA-DCLS-1404/2020 & Cys488 & Arg & /IRN/COVID19-IRVSH4/2020 \\
\hline & Arg & /USA/CA-CZB-12872/2020 & \multirow{2}{*}{ Phe 490} & Leu & $\begin{array}{l}\text { /AUS/NIC10024/2020 } \\
\text { /AUS/VIC766/2020 }\end{array}$ \\
\hline & \multirow{2}{*}{ Met } & \multirow{2}{*}{\begin{tabular}{|l} 
/BHR/340798279_S5_L001/2020 \\
/USA/CA-CZB-1043/2020 \\
/IND/906/2020 \\
\end{tabular}} & & Val & /USA/MA-MGH-01415/2020 \\
\hline & & & Pro491 & His & $/ / \mathrm{ND} / 906 / 2020$ \\
\hline Leu455 & Phe & $\begin{array}{l}\text { /AUS/VIC10121/2020 } \\
\text { /AUS/VIC5196/2020 }\end{array}$ & $\mathrm{G} \ln 493$ & Leu & /USA/WI-UW-371/2020 \\
\hline \multirow[t]{2}{*}{ Val483 } & Ala & $\begin{array}{l}\text { /USA/WA-UW-6527/2020 } \\
\text { /USA/WA-UW-1587/2020 } \\
\text { /USA/WA-RML-2/2020 } \\
\text { /USA/WA-RML-6/2020 } \\
\text { /USA/WA-RML-5/2020 } \\
\text { /USA/UT-03764/2020 } \\
\text { /AUS/VIC2139/2020 }\end{array}$ & \multirow[t]{2}{*}{ Ser494 } & Pro & $\begin{array}{l}\text { /USA/CA-CZB-4047/2020 } \\
\text { /USA/CA-CZB-11677/2020 } \\
\text { /USA/CA-CZB-6994/2020 } \\
\text { /USA/CA-CZB-11010/2020 } \\
\text { /USA/MI-MDHHS-SC20047/2020 } \\
\text { /USA/CA-CZB-12810/2020 } \\
\text { /AUS/NIC9505/2020 }\end{array}$ \\
\hline & Phe & $\begin{array}{l}\text { /AUS/NIC2139/2020 } \\
\text { /USA/MA-UW-629/2020 }\end{array}$ & & Leu & /AUS/VIC5057/2020 \\
\hline \multirow{3}{*}{ Glu484 } & Gln & \begin{tabular}{|l} 
/USA/UT-UPHL-2009538/2020 \\
/IND/GBRC278a/2020 \\
/USA/SEARCH-1462-SAN/2020
\end{tabular} & & & \\
\hline & Lys & $\begin{array}{l}\text { /USA/UT-QDX-1869/2020 } \\
\text { /BHR/340859913_S11/2020 } \\
\text { /USA/LLUW-379/2020 }\end{array}$ & & & \\
\hline & Ala & /USA/VA-DCLS-1615/2020 & & & \\
\hline
\end{tabular}

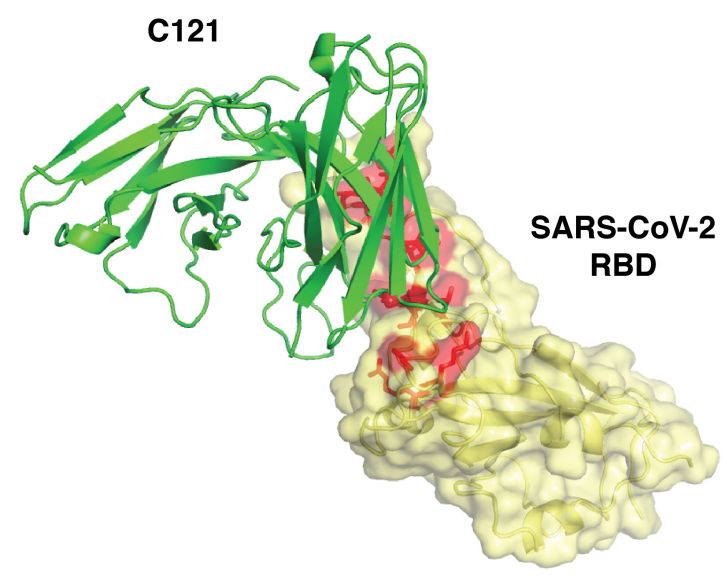

\begin{tabular}{|c|c|c|}
\hline wt & mutant & Strain Name \\
\hline Phe342 & Leu & England/01, 1/29 \\
\hline \multirow{3}{*}{ Ala344 } & Ser & $\begin{array}{l}\text { /USA/WA-S2278/2020 } \\
\text { /USA/WA-S2530/2020 }\end{array}$ \\
\hline & Thr & /IND/GBRC431a/2020 \\
\hline & Val & /AUS/NIC10958/2020 \\
\hline \multirow{2}{*}{ Thr345 } & Ser & /USA/WA-S1049/2020 \\
\hline & Ile & /PER/covper051/2020 \\
\hline Arg346 & Thr & /IND/GBRC333/2020 \\
\hline Trp436 & Thr & /IND/GBRC333/2020 \\
\hline Asn439 & Lys & /USA/IL-UW/799/2020 \\
\hline Asn440 & Lys & /HKG/Case5138/2020 \\
\hline Leu441 & Ile & /USA/FL-BPHL-0297/2020 \\
\hline Asn450 & Lys & $/ / \mathrm{ND} / 906 / 2020$ \\
\hline
\end{tabular}

Extended Data Fig. 8 | Natural SARS-CoV-2 variants in the $\mathrm{C121}$ and $\mathrm{C135}$ epitopes. a, b, Summary of naturally occurring mutations in the $\mathrm{C} 121$ (a) or $\mathrm{C} 135$ (b) epitopes that have been reported in circulating SARS-CoV-2 (as of 1January 2021). The location of the mutated residues is shown in red on the RBD

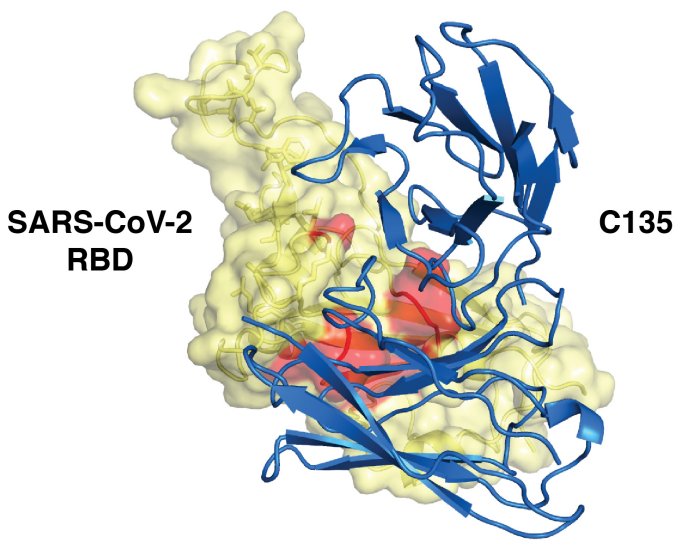

structure. The $\mathrm{C} 121$ and $\mathrm{C} 135$ variable regions are in green and blue, respectively (PDB 7K8X and 7K8Z, respectively). All the variants were taken from the ViPR database (https://www.viprbrc.org/). 
Extended Data Table 1 | Summary of the $P$ values for the mouse protection experiment

\begin{tabular}{r|c|c|c|c} 
& $\mathbf{C 1 2 1}$ & $\mathbf{C 1 3 5}$ & $\mathbf{C o V}-\mathbf{X 2}$ & Isotype control \\
\hline $\mathbf{C 1 2 1}$ & - & $\mathrm{P}<0.0001$ & $\mathrm{P}<0.0001$ & $\mathrm{P}<0.01$ \\
$\mathbf{C} 135$ & $\mathrm{P}<0.0001$ & - & $\mathrm{P}>0.05$ & $\mathrm{P}<0.0001$ \\
$\mathbf{C o V}-\mathbf{X 2}$ & $\mathrm{P}<0.0001$ & $\mathrm{P}>0.05$ & - & $\mathrm{P}<0.0001$ \\
Isotype control & $\mathrm{P}<0.01$ & $\mathrm{P}<0.0001$ & $\mathrm{P}<0.0001$ & -
\end{tabular}

Statistical comparison of body weight differences in mice treated with the individual monoclonal antibodies (C121 or C135), the CoV-X2 bispecific antibody or isotype control at $8 \mathrm{dpi}$ (data shown in Fig. 2e). $P$ values were determined with a one-way analysis of variance (ANOVA). Comparison of the entire curves (Fig. 2e) by the one-sample Wilcoxon test or by ANOVA followed by Turkey-Kramer post test reveals that the isotype-control-treated group is statistically different from any one of the other groups (CoV-X2, $P=0.0159 ; \mathrm{C} 135, P=0.0043$; and C121, $P=0.0010)$. 


\section{Reporting Summary}

Nature Research wishes to improve the reproducibility of the work that we publish. This form provides structure for consistency and transparency in reporting. For further information on Nature Research policies, see our Editorial Policies and the Editorial Policy Checklist.

\section{Statistics}

For all statistical analyses, confirm that the following items are present in the figure legend, table legend, main text, or Methods section.

n/a Confirmed

$\square$ The exact sample size $(n)$ for each experimental group/condition, given as a discrete number and unit of measurement

$\square$ A statement on whether measurements were taken from distinct samples or whether the same sample was measured repeatedly

The statistical test(s) used AND whether they are one- or two-sided

$\square$ Only common tests should be described solely by name; describe more complex techniques in the Methods section.

Х $\square$ A description of all covariates tested

Х $\square$ A description of any assumptions or corrections, such as tests of normality and adjustment for multiple comparisons

$\triangle$ A full description of the statistical parameters including central tendency (e.g. means) or other basic estimates (e.g. regression coefficient)

$\bigotimes$ AND variation (e.g. standard deviation) or associated estimates of uncertainty (e.g. confidence intervals)

$\varnothing$ For null hypothesis testing, the test statistic (e.g. $F, t, r$ ) with confidence intervals, effect sizes, degrees of freedom and $P$ value noted Give $P$ values as exact values whenever suitable.

Х $\square$ For Bayesian analysis, information on the choice of priors and Markov chain Monte Carlo settings

Х $\square$ For hierarchical and complex designs, identification of the appropriate level for tests and full reporting of outcomes

Х $\square$ Estimates of effect sizes (e.g. Cohen's $d$, Pearson's $r$ ), indicating how they were calculated

Our web collection on statistics for biologists contains articles on many of the points above.

\section{Software and code}

Policy information about availability of computer code

Data collection SPR data were collected with Biacore 8K Control Software v2.0.15.12933.

ELISA plates were measured with the reader software Gen5 Version 1.11.5, BioTek Instruments, Inc.

DLS measurements were performed using Dynamics v7.1.7.16, Wyatt Tecnology Corp.

The building of bispecific antibody was performed using ALMOST toolkit version 1 and Pymol 2.4.

The Molecular Dynamics simulations were performed using Gromacs 2020.2.

LightCycler ${ }^{\circledR} 480$ Software, Version 1.5

Modulus II Microplate Reader User interface version 2.1.0 by TURNER BioSystems

I-TASSER Suite 5.1

Image Jersion $1.53 \mathrm{~h}$

Data analysis SPR data were analyzed with Biacore Inside Evaluation Software v2.0.15.12933.

DLS data were analyzed using Dynamics v7.1.7.16 (Wyatt Tecnology Corp.)

Data from ELISA and mouse experiments were analyzed with GraphPad Prism Version 8.4.2.

The 3D structures were analyzed using Pymol 2.4.

The MD trajectories were analyzed using Gromacs 2020.2

Microsoft Excel 2016

BioEdit Sequence Alignment Editor, version 7.2.0. 
Policy information about availability of data

All manuscripts must include a data availability statement. This statement should provide the following information, where applicable:

- Accession codes, unique identifiers, or web links for publicly available datasets

- A list of figures that have associated raw data

- A description of any restrictions on data availability

The authors declare that data supporting the findings of this study are available within the paper and its supplementary information files. All other data are available from the corresponding author upon reasonable request. Published data were taken from GenBank (https://www.ncbi.nlm.nih.gov/genbank/), UniProt (https:// www.uniprot.org/), Protein Data Bank, PDB (https://www.rcsb.org/) and ViPR database (https://www.viprbrc.org/).

\section{Field-specific reporting}

Please select the one below that is the best fit for your research. If you are not sure, read the appropriate sections before making your selection. \Life sciences Behavioural \& social sciences Ecological, evolutionary \& environmental sciences

For a reference copy of the document with all sections, see nature.com/documents/nr-reporting-summary-flat.pdf

\section{Life sciences study design}

All studies must disclose on these points even when the disclosure is negative.

Sample size No sample-size calculation was performed. The sample sizes were chosen based on experience and previously published papers (e.g., Zost et al., Nature. 2020 Aug;584(7821):443-449. doi: 10.1038/s41586-020-2548-6.; Hassan et al., Cell. 2020 Aug 6;182(3):744-753.e4. doi: 10.1016/ j.cell.2020.06.011.). Details about groups and sample sizes for mouse virus challenge studies are provided in the manuscript and figure legends.

Data exclusions No data were excluded.

Replication Experiments successfully repeated at least twice.

Randomization The mice were randomly assigned to cages and the cages were then randomized into groups.

Blinding Blinding was not relevant to this study, except lung pathology evaluation. The readouts of all experiments, except lung pathology evaluation, could be assessed objectively. Mouse weight loss was determined using body weight measurement as a readout, and plaque assay or RT-qPCR was used to quantify viral burden. For lung study pathology, H\&E stained tissue sections were scored by an experienced histopathologist blinded to the compositions of the groups.

\section{Reporting for specific materials, systems and methods}

We require information from authors about some types of materials, experimental systems and methods used in many studies. Here, indicate whether each material, system or method listed is relevant to your study. If you are not sure if a list item applies to your research, read the appropriate section before selecting a response.

Materials \& experimental systems

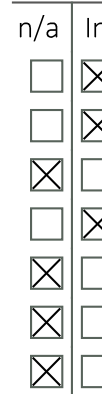

\begin{tabular}{|c|c|}
\hline \multicolumn{2}{|c|}{ Methods } \\
\hline $\mathrm{n} / \mathrm{a}$ & Involved in the study \\
\hline X & $\square$ ChIP-seq \\
\hline Х & $\square$ Flow cytometry \\
\hline$\bigotimes$ & $\square$ MRI-based neuro \\
\hline
\end{tabular}

Animals and other organisms

$\searrow \square$ Human research participants

Х $\square$ Clinical data

$\bigotimes \square$ Dual use research of concern

\section{Antibodies}

Antibodies used

Primary antibodies used:

- In this study we used and analyzed antibodies C121, C135 and C144 against SARS-CoV-2 (in house production).

- Anti-Zika virus monoclonal antibody Z021 (Robbiani et al, Cell 2017) used as isotype control

- Anti-Histone H3 antibody, Cat. No.: ab1791 (Abcam), lot: GR3237685-2, dilution: 1:1000

- Anti-ACE2 antibody, Cat. No.: ab15348 (Abcam), lot: GR3333640-7, dilution: 1:1000

- F4/80 (D2S9R) XP ${ }^{\circledR}$ Rabbit mAb (Cat.\#70076) Cell Signaling Technology, USA, Lot 5, RRID AB_2799771, dilution 1:800 
Following secondary antibodies were used:

- Goat Anti-Mouse IgG, Human ads-AP, Cat.-No.: 1030-04 (SouthernBiotech, dilution 1:500)

- Goat Anti-Human IgG-AP, Cat.-No.: 2040-04 (SouthernBiotech, dilution 1:500)

- HRP-Polymer anti-Rabbit (Ready-to-use), Zytomed, Germany, Cat. \# ZUC 032-100, Lot A0880-4 (no dilution)

- Goat Anti-Rabbit IgG H\&L (HRP), Cat. No.: ab205718 (Abcam), lot: GR3269880-1, dilution: 1:10000

Validation

The human monoclonal antibody Z021, which binds to the Envelope Domain III of the Zika virus, was previously reported and validated (PMID: 31413072).

No validation statements for the other antibodies that are commercially available.

\section{Eukaryotic cell lines}

Policy information about cell lines

Cell line source(s)

Expi293F cells (Thermo Fisher Scientific)

293 [HEK-293] cell line (CRL-1573 ${ }^{\text {TM }}$ ) (ATCC ${ }^{\circledR}$ )

293AAV cell line (AAV-100) (Cell Biolabs, Inc)

Neuro-2a cell line (CCL-131 ${ }^{\mathrm{TM}}$ ) (ATCC $\left.{ }^{\circledR}\right)$

Vero E6 (CRL-1586 ${ }^{\mathrm{TM}}$ ) (ATCC ${ }^{\circledR}$ )

293TAce2 (derived from 293T as reported in Robbiani et al. Nature, DOI: 10.1038/s41586-020-2456-9)

Authentication

Not authenticated after purchase

Mycoplasma contamination

In situ analysis never detected Mycoplasma infection.

Commonly misidentified lines

(See ICLAC register)

No commonly misidentified cell line have been used in this study.

\section{Animals and other organisms}

Policy information about studies involving animals; ARRIVE guidelines recommended for reporting animal research

Laboratory animals Mice C57BI/6NCrl (females, 13-15 weeks old). Mice were housed in individually ventilated cages (Green line, Tecniplast, Italy) in the controlled environment with a $12 / 12$ hour light-dark cycle, $20-22{ }^{\circ} \mathrm{C}$ and $45-65 \%$ humidity. Animals were fed the standard chow diet (1314 Altromin, Germany) ad libitum. Drinking water was filtered by reverse osmosis and chlorinated to 1.5 ppm. Water bottles were changed weekly.

Wild animals

The study did not involve wild animals.

Field-collected samples

The study did not invovle field-collected samples.

Ethics oversight

The experiments were approved by the Committee on the Ethics of Animal Experiments of the Institute of Parasitology, Biology Centre of the Czech Academy of Sciences, Institute of Molecular Genetics of the Czech Academy of Sciences, and of the Departmental Expert Committee for the Approval of Projects of Experiments on Animals of the Academy of Sciences of the Czech Republic (permits 82/2020 and 101/2020).

Note that full information on the approval of the study protocol must also be provided in the manuscript. 